\title{
Quantum-ring spin interference device tuned by quantum point contacts
}

\author{
Leo Diago-Cisneros ${ }^{1}$ and Francisco Mireles $^{2}$ \\ ${ }^{1}$ Facultad de Física, Universidad de La Habana, \\ C.P.10400, La Habana, Cuba and \\ ${ }^{2}$ Centro de Nanociencias y Nanotecnología, \\ Universidad Nacional Autónoma de México, \\ C.P. 22800 Ensenada, Baja California, México.
}

(Dated: June 8, 2022)

\begin{abstract}
We introduce a spin-interference device that comprises a quantum ring (QR) with three embedded quantum point contacts (QPCs) and study theoretically its spin transport properties in the presence of Rashba spin-orbit interaction. Two of the QPCs conform the lead-to-ring junctions while a third one is placed symmetrically in the upper arm of the QR. Using an appropriate scattering model for the QPCs and the $\mathbb{S}$-matrix scattering approach, we analyze the role of the QPCs on the Aharonov-Bohm (AB) and Aharonov-Casher (AC) conductance oscillations of the QR-device. Exact formulas are obtained for the spin-resolved conductances of the QR-device as a function of the confinement of the QPCs and the $\mathrm{AB} / \mathrm{AC}$ phases. Conditions for the appearance of resonances and anti-resonances in the spin-conductance are derived and discussed. We predict very distinctive variations of the QR-conductance oscillations not seen in previous $\mathrm{QR}$ proposals. In particular we find that the interference pattern in the $\mathrm{QR}$ can be manipulated to a large extend by varying electrically the lead-to-ring topological parameters. The latter can be used to modulate the $\mathrm{AB}$ and $\mathrm{AC}$ phases by applying gate voltage only. We have shown also that the conductance oscillations exhibits a crossover to well-defined resonances as the lateral QPC confinement strength is increased, mapping the eigenenergies of the QR. In addition, unique features of the conductance arises by varying the aperture of the upper-arm QPC and the Rashba spin-orbit coupling. Our results may be of relevance for promising spin-orbitonics devices based in quantum interference mechanisms.
\end{abstract}




\section{INTRODUCTION}

Quantum rings structures not only provides an excellent physics playground to study coherent transport and electronic interference phenomena, but they also constitutes very appealing systems towards the realization of novel spintronic[1] devices. The AharonovBohm[2] (AB) effect and its relativistic counterpart, the Aharonov-Casher [3] (AC) effect, are just two of these quantum interference phenomena that may appear simultaneously in semiconductor quantum rings. They manifest as an oscillatory behavior of the conductance of the quantum ring, either as a magnetic flux is varied (AB-effect), or as a function of the spin-orbit interaction (SOI) strength (AC-effect) in the semiconductor ring. In the AB-effect, is the accumulated phase acquired by two electronic waves traveling coherently around a close loop in opposite directions that leads to magnetoconductance oscillations. In contrast, in the AC-effect, is the precessing intrinsic magnetic moment (spin) of the electrons winding the ring, that acquires a phase change due to a gate-voltage tunable SOI. [4-7] As a result, periodic conductance oscillations can be observed as a perpendicular external electric field is varied. [8]

The AB-effect was first observed by Webb et al. in metallic rings. [9] A few years later Cimmino et al. [10] performed experiments using neutron (spin 1/2) beams following a closed path observing signals of the AC-oscillation phenomena. The AC-phase oscillations has been observed also in a $\mathrm{HgTe} / \mathrm{HgCdTe}$ based single quantum ring[1] and in small arrays of mesoscopic InGaAs/InAlAs based rings [12] which exhibit strong Rashba-SOI. 13]

Additional phase effects concern the Berry (geometric) phase, acquired by a quantal particle traveling around a circuit in inhomogeneous effective magnetic fields.[14] Berry phase signatures in the conductance has been studied in one- and two-dimensional (2D) rings with Rashba SOI.[15, 16] More recently the AC-phase oscillations were measured in an array of gated InGaAs/InAlAs-based quantum rings as a function of the Rashba SOI strength and of the rings radius.[17] Interestingly, the authors were also able to observe a topological (geometric) spin phase, [18] that together with its dynamical phase, contributes to the overall time-reversal AC-phase change. [17, 19]

Micron-sized and nanoscale semiconductor quantum rings fabricated on two-dimensional electron gases (2DEG) are the base of several spintronics proposal devices, ranging from spininterference devices that modulate the output electric current, [20, 21] spin-filters, [22, 23] 
and quantum splitters, 24] to mention a few.

In this work we propose a spin-interference device setup composed by a semiconductor quantum-ring (QR) with Rashba-SOI at the center of the structure, and connected to a source and drain of electrons via quantum point contacts (QPCs). In contrast to previous studies, the ring junctions (here characterized by QPCs) are allocated symmetrically, just at the QR periphery. Additionally a third QPC is located in one of the arms of the QR device (Fig, 1). Most notably, all QPCs are presumed to be 2D and independently tuned with in-plane electrical gates. By doing so, we additionally manage to overcome several disadvantages of former related studies that relied on a free parameter $\epsilon$ to characterize the QR-coupling with the leads. [21, 26, 27]

As far we know, a study of the role of QPCs with variable electrostatic width, allocated at the inlet and outlet of a quantum ring (QR), has not been reported before, neither experimentally, nor theoretically. Same applies for the case of a QR-device with an inserted QPC with tunable width at one of the arms of the QR. Hence just the proposal itself constitutes an interesting idea worth of a detailed study. Is very important to remark also that this setup offers the possibility of tuning the spin-interference phenomenon in the QR just by controlling electrostatically the effective width of the QPCs, in the presence or not, of an external magnetic fields as we shall see later.

Here we study theoretically the coherent electronic quantum transport and the spin interference in the described QR-based setup above in the presence of Rashba-SOI, and investigate the role of the lead-to-ring junctions via QPCs together with an upper-arm QPC. The backscattering and tunneling -through the lead-to-ring junctions- were modeled by two-dimensional saddle point potentials describing here the QPC electrical confinement. Following Büttiker[25] and Vasilopoulus et al.[27] an appropriate scattering matrix $\mathbb{S}$ formalism is established. Invoking then time-reversal invariance and unitarily relations of the $\mathbb{S}$ matrix we are able to derive the relevant tunneling quantities through the whole structure. Exact formulas for the quantum conductance of the QR-device are obtained. We find that two-probe conductance oscillations of the ring are rather sensitive to the confinement strength at the QPCs, as well as with the applied magnetic field and the spin orbit interaction intensity. We predict distinctive variations of the two-probe QR-conductance oscillations which can be used to modulate the Aharonov-Bohm and Aharonov-Casher phases by applying gate voltages. It is shown as well that is possible to control interference pattern 
in the QR to a large extend by varying the lead-to-ring topological parameters. Our results might be therefore of relevance for promising spin-orbitonics [19] devices based in quantum interference mechanisms.

The remainder of the paper is organized as follows. In Section [II we introduce the quantum ring device under study as well as the QPC model employed. In Section [II we analyze the bare quantum ring Hamiltonian and its eigensolutions. The $\mathbb{S}$-matrix formalism applied to the whole QR-device is presented in Section IV. The main formulas of this paper are derived in this section. In Section $\nabla$ we discuss in detail several illustrative numerical simulations of the spin interference properties occurring in the proposed device configuration. A Summary is provided in Section VI.

\section{QUANTUM RING DEVICE PROPOSAL AND THE 2D-QPC MODEL}

\section{A. Quantum ring device geometry}

Consider a quantum ring of radius $R$ and width $w$ defined electrostatically on a semiconductor 2DEG through some radial confining potential. It can be produced e.g. by applying a gate voltage to a metallic ring-shape structure deposited on top of the semiconductor heterostructure containing the 2DEG. Fig. 1 1 shows a sketch of a top view of the whole device configuration envisioned here. We assume that the two-probe device consist of the $\mathrm{QR}$ at the center of the structure connected to two leads by QPCs conforming the lead-to-ring junctions (interfaces). The leads are considered in ohmic contact with a QPC/QR*/QPC structure, where $\mathrm{QR}^{*}$ denotes here that an additional QPC has been inserted in the upper arm of the QR. The effective widths (transparency) of all QPCs are assumed to be gate-tunable in an independent manner.

Theoretically, the role of the electron scattering at the junctions lead-to-ring on the Aharonov-Bohm oscillations was first addressed by Büttiker 25] by studying metallic QRs within the $\mathbb{S}$-matrix formalism. The approach has been employed subsequently to model transport in semiconductor AB-rings as well as AC spin-orbit effects. [21, 23, 27] Despite its versatility, the approach relies on a free parameter ( $\epsilon$ in Ref.[25]) used to characterize the QR-coupling with the leads, and modeled as a point-like scatterers. The free parameter $\epsilon$ represents the transmission amplitude from one lead to the $\mathrm{AB}$ ring, or vice versa, and can 


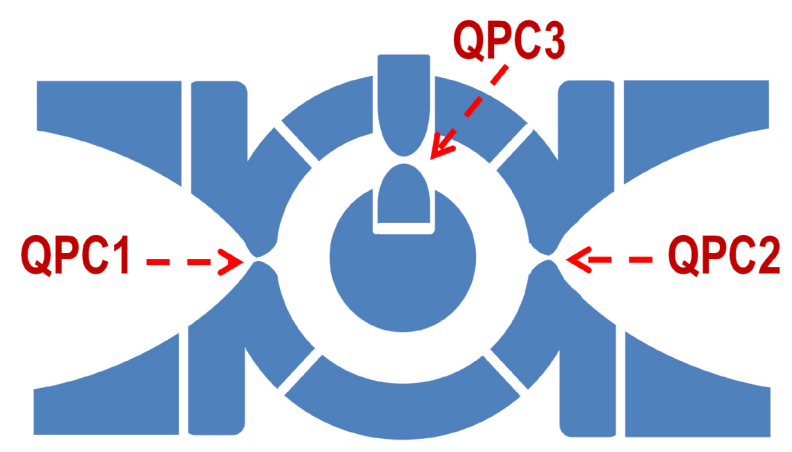

FIG. 1: (Color online) Schematic diagram showing a top view of the envisioned QR device. Two quantum point contacts $\left(\mathrm{QPC}_{1,2}\right)$ are allocated symmetrically outside the $\mathrm{QR}$ path, just at the QR periphery. An additional $\mathrm{QPC}_{3}$ is considered in the $\mathrm{QR}$ upper arm. All QPCs are supposed to be controlled independently by in-plane gate voltages.

take values in the range $0 \leq \epsilon \leq 1 / 2$; where perfect reflection means $\epsilon=0$, while perfect transmission entails $\epsilon=1 / 2$. The introduction of this parameter was a first effort to describe the possible band mismatch between the leads and the conducting AB-QR. Furthermore the model introduces a second caveat, which arises when a third scatterer is considered in one of the arms of the QR. It predicts the counterintuitive result of a finite emerging current flux form the QR (non-zero electron transmission probability) in the limit case of a zero dynamic phase change $\left(\theta=\pi R \sqrt{2 m E_{\mathrm{F}} / \hbar^{2}}\right)$, i.e. eventhough the incident Fermi energy $E_{\mathrm{F}}$ is set to zero and there is no bias voltage present. [23, 27] Clearly a special care have to be exercised when modeling the lead-to-ring for the case of semiconductor junctions.

Here we propose to replace the point-like scatterers that model the lead-to-ring junctions by two-dimensional quantum point contacts instead. By doing so, the dependence on a free parameter is naturally avoided in the model, and very important, the proposed geometry give us additional degrees of freedom to manipulate the conductance and spin interference in the $\mathrm{QPC} / \mathrm{QR} * / \mathrm{QPC}$ device.

As we will show later, the conductance quantum oscillations in the $\mathrm{QPC} / \mathrm{QR} / \mathrm{QPC}$ device can be related not only to magnetic flux and the SOI effects as in earlier reports, [23, 27] but also to the confinement strength of the QPCs. Indeed, this new feature (tunability of the QPCs) we have considered in the QR device allows means to control the spin-conductance oscillations of the output current, and in turn, can be used to modulate the Aharonov-Bohm 
and Aharanov-Casher phases solely by applying gate voltages. Furthermore, it is predicted that the insertion of tunable QPCs in the QR leads to new and distinguishable physical response of the conductance oscillations of the QR, which are in principle, measurable at low temperatures. Hence a rich variety of interference phenomena arises which may be also of interest for potential applications.

\section{B. Quantum point contact model}

The lead-to-ring junctions of the device are assumed to form a narrow quantum constriction, i.e., a QPC. Each QPC (including the one at the upper arm of the QR) are modeled through saddle point electrostatic potentials whose confinement are in principle tunable by external gates. To a good approximation the QPC can be represented through a purely quadratic two-dimensional potential,

$$
V_{\mathrm{SP}}(x, y)=V_{o}-\frac{1}{2} m \omega_{x}^{2} x^{2}+\frac{1}{2} m \omega_{y}^{2} y^{2} .
$$

where $V_{o}$ is the potential energy at the saddle point, $m$ is the effective mass of the conduction electrons, and $\hbar \omega_{x}$ and $\hbar \omega_{y}$, are the characteristic longitudinal and the transversal energy confinements, respectively. The reference energy $V_{o}$ as well as the lateral $x-y$ confinement, can be adjusted in the actual experiments through electrical gates in an in-

dependent way. Remarkably, as deduced by Fertig and Halpering, [28] in the presence of a magnetic flux - due to a perpendicular magnetic field $B$ - the electron transmission amplitude of the two-dimensional potential (1) can be described solely, by a dimensionless parameter $\varepsilon$, which is a function of all the relevant energies characterizing the QPC. Namely $\varepsilon=\varepsilon\left(E_{\mathrm{F}}, \omega_{x}, \omega_{y}, \omega_{c}\right)$, where $\omega_{c}=e B / m c$ is the cyclotron frequency. The quantum transmission amplitude reads, 28,

$$
t_{s}(\varepsilon)=\frac{1}{2}\left(\frac{\Gamma\left(\frac{1}{4}-\frac{1}{2} i \varepsilon\right)}{\Gamma\left(\frac{1}{4}+\frac{1}{2} i \varepsilon\right)} e^{i \pi / 4}-\frac{\Gamma\left(\frac{3}{4}-\frac{1}{2} i \varepsilon\right)}{\Gamma\left(\frac{3}{4}+\frac{1}{2} i \varepsilon\right)} e^{-i \pi / 4}\right),
$$

where $\Gamma(z)$ is the Gamma function. The transmission probability of the QPC, $T_{s}=\left|t_{s}\right|^{2}$ simplifies to

$$
T_{s}(\varepsilon)=\frac{1}{1+\exp (-\pi \varepsilon)},
$$

which satisfies $T_{s}(\varepsilon)+R_{s}(\varepsilon)=1$, and thus

$$
R_{s}(\varepsilon)=\frac{1}{1+\exp (\pi \varepsilon)}
$$


will be the reflection probability that an incoming electron with unitary amplitude has been elastically backscatter from the QPC. In the limit $|\varepsilon| \gg 1$, we have for $\varepsilon>0, T_{s}(\varepsilon) \approx 1-e^{-\pi \varepsilon}$,

while for $\varepsilon<0$, we have $T_{s}(\varepsilon) \approx e^{-\pi|\varepsilon|}$. Explicitly, the parameterized energy is given by $\varepsilon=\left(E_{G}-V_{o}\right) / E_{1}$, and give us a measure of the energy of the semi-classical guiding-center motion $E_{G}$ relative to $V_{o}$. Here $E_{G}=E-\left(n+\frac{1}{2}\right) E_{2}$, with $n$ (non-negative integer) denoting the quantum index for the quasi-Landau levels. The energies $E_{\nu}$ with $\nu=1,2$ are given explicitly by

$$
E_{\nu}=\frac{\nu \hbar}{2 \sqrt{2}} \sqrt{\sqrt{\Omega^{4}+4 \omega_{x}^{2} \omega_{y}^{2}}+(-1)^{\nu} \Omega^{2}},
$$

with $\Omega^{2}=\omega_{c}^{2}+\omega_{y}^{2}-\omega_{x}^{2}$. Since in the limit of $\omega_{x, y} \ll \omega_{c}, E_{2} \approx \hbar \omega_{c}$, then $\Omega$ is called the effective oscillator frequency. 28] Semi-classically, a charge carrier entering a region crossed by a magnetic field $B$, perpendicular to 2DEG and to the QR plane, will describe a circular motion with cyclotron frequency $\omega_{c}$. It is clear that the intensity of the scattering magnitudes (2) is directly bonded to $E_{G}$. Therefore, one can distinguish two different scenarios for the electronic transport, namely: (i) quantum tunneling for $E_{G}<V_{o}$, and (ii) quantum transmission following quasi-classical motion around the saddle-point potential for $E_{G}>V_{o}$. Here we will be interested in the latter case, with the cyclotron frequency written in terms of the magnetic flux penetrating the QR as $\omega_{c}=(e / \pi m c) \Phi / R^{2}$, where $\Phi=\pi R^{2} B$ is the magnetic flux.

Before we go into the details of the scattering process in the QPC/QR*/QPC device, it is convenient to revisit first the Hamiltonian model used to model the AB-AC-QR without scatterer (QPCs) and in presence of Rashba-SOI.

\section{AB-AC QUANTUM RING HAMILTONIAN WITH SOI}

The single-electron two-dimensional Hamiltonian for a semiconductor quantum ring in the presence of Rashba-SOI (without scatterers) and in the absence of a magnetic field is first described in polar cylindrical coordinates. It reads

$$
H(r, \varphi)=H_{o}(r)+H^{\prime}(r, \varphi)
$$

where the purely radial term is given by 29$]$

$$
H_{o}(r)=-\frac{\hbar^{2}}{2 m}\left(\frac{\partial^{2}}{\partial r^{2}}+\frac{1}{r} \frac{\partial}{\partial r}\right)+V(r),
$$


whereas the angular dependence as well as the Rashba-SOI term, is contained in

$$
H^{\prime}(r, \varphi)=-\frac{\hbar^{2}}{2 m r^{2}} \frac{\partial^{2}}{\partial \varphi^{2}}-i \alpha_{s o} \boldsymbol{\sigma} \cdot\left(\hat{\boldsymbol{e}}_{r} \frac{1}{r} \frac{\partial}{\partial \varphi}-\hat{\boldsymbol{e}}_{\varphi} \frac{\partial}{\partial r}\right)
$$

where $\alpha_{s o}$ stands for the Rashba-SOI parameter strength, the matrix vector $\boldsymbol{\sigma}=\left(\sigma_{x}, \sigma_{y}, \sigma_{z}\right)$, being $\sigma_{x, y, z}$, the usual spin Pauli matrices, $\hat{\boldsymbol{e}}_{r}=(\cos \varphi, \sin \varphi, 0)$ and $\hat{\boldsymbol{e}}_{\varphi}=(-\sin \varphi, \cos \varphi, 0)$ are unitary vectors along the radial and azimuthal direction, respectively. Following Meijer et al., [29] in the limit of a narrow ring (i.e. strong radial confinement), the associated confining energy in the radial direction becomes (typically) much larger than the characteristic SOI and kinetic energies of the circling electrons around the ring. Thus $H^{\prime}(r, \varphi)$ can be treated perturbatively by introducing an appropriate model potential $V(r)$ by solving first the purely radial term $H_{o}(r)$. An effective one-dimensional (1D) Hamiltonian depending only on the azimuthal angle $\varphi$ can be obtained by taking the expectation value of Eq.(7) with the eigenfunctions of the Hamiltonian $H_{o}$ in the lowest radial mode, $H_{1 D}(\varphi)=\left\langle R_{o}(r)\left|H^{\prime}(r, \varphi)\right| R_{o}(r)\right\rangle$. The effective 1D Hamiltonian takes the form [29, 30]

$$
H_{1 D}=\frac{\hbar^{2}}{2 m R^{2}}\left\{\left(i \frac{d}{d \varphi}+k_{s o} R\left(\boldsymbol{\sigma} \cdot \hat{\boldsymbol{e}}_{\varphi}\right)\right)^{2}-\left(k_{s o} R\right)^{2}\right\},
$$

with $k_{s o}=m \alpha_{s o} / \hbar^{2}$. Then to first order, we can rewrite (5) as

$$
H(r, \varphi)=H_{o}(r)+H_{1 D}(\varphi),
$$

as a separable in $r$ and $\varphi$ Hamiltonian to deal with.

Up to this point we have not considered yet the effect of an external magnetic field (magnetic flux) across the path of the winding electrons in the ring. This is done by introducing the usual minimal coupling substitution of the momentum operator, $-i \nabla \rightarrow-i \nabla-e \boldsymbol{A}$. The vector potential can be conveniently chosen to be tangential to the QR for a magnetic field $B$ perpendicular to the plane of the ring by using $\boldsymbol{A}=(B R / 2) \hat{\boldsymbol{e}}_{\varphi}$. As a result the moving electrons flowing around the ring will experiment a magnetic flux given by $\Phi=\oint \boldsymbol{A} \cdot\left(R d \varphi \hat{\boldsymbol{e}}_{\varphi}\right)=\pi R^{2} B$. This is formally equivalent to the replacement $i d / d \varphi \rightarrow i d / d \varphi+\Phi / \Phi_{o}$ in Eq.(8), where $\Phi_{o}=h / e$ is the the magnetic flux quanta. The latter gives rise to the well known $\mathrm{AB}$ phase $\left(\Phi_{A B}=2 \pi \Phi / \Phi_{o}\right)$ acquired by the electrons moving in the close path of the quantum in the presence of a magnetic flux. As a consequence the physical information of $\mathrm{AB}$ phase can be carried out directly in the overall phase of the eigenstates of the SOI 1D-Hamiltonian (8). [30, 31] Note that the magnetic field may 

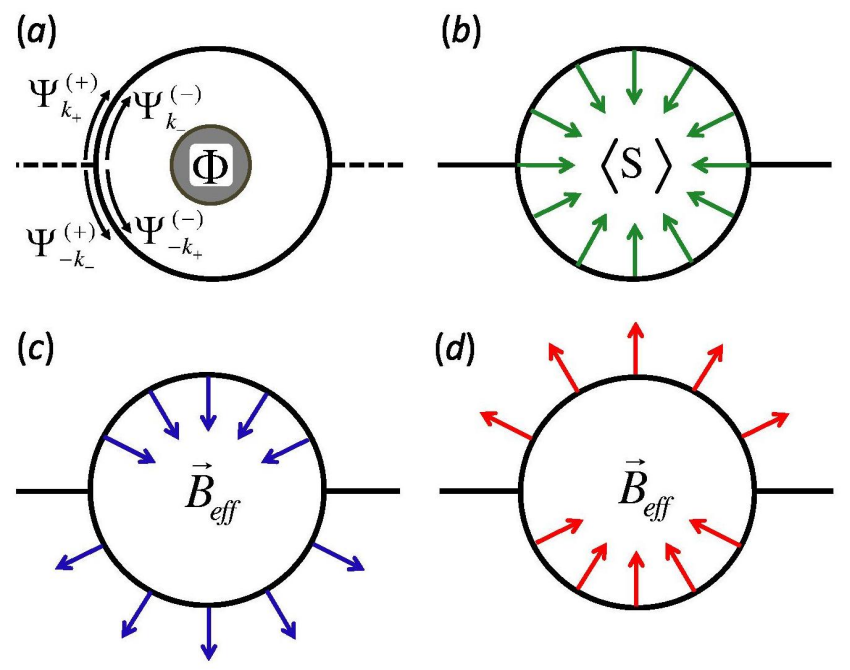

FIG. 2: (Color online) (a) The diagram sketches the clockwise and anti-clockwise propagation for the two spin-resolved eigenstates, defined in 10 and 110 within the adiabatic approximation. (b) Spin orientation $\langle\boldsymbol{S}\rangle$ of the electrons winding the ring. (c) and (d) Rashba induced effective magnetic field $\boldsymbol{B}_{\text {eff }}$ for clockwise and anti-clockwise cycling electrons, respectively.

or not penetrate the QR. Here we are interested in the relatively weak magnetic field regime, or either the case in which just the magnetic flux penetrates the AB-AC-QR (see Fig,2a), but not the field itself, and therefore the Zeeman effect can be safely neglected in the full description.

Given that the projection of the total angular momentum along the $z$-axis commutes with the 1D Hamiltonian, $\left[J_{z}, H_{1 D}\right]=0$, with $J_{z}=-i \hbar(\partial / \partial \varphi)-\hbar \sigma_{z} / 2$. Then it follows that a particular set of eigensolutions of the system $H_{1 D}|\Psi(\varphi)\rangle=E|\Psi(\varphi)\rangle$ per spin orientation and clockwise/anticlockwise $(+/-)$ propagation of the electrons can be written as [23, 24]

$$
\begin{aligned}
& \left|\Psi_{k_{+}}^{(+)}\right\rangle=e^{i k_{+} R \varphi}|+\rangle, \quad\left|\Psi_{k_{-}}^{(+)}\right\rangle=e^{-i k_{-} R \varphi}|+\rangle, \\
& \left|\Psi_{k_{-}}^{(-)}\right\rangle=e^{i k_{-} R \varphi}|-\rangle, \quad\left|\Psi_{k_{+}}^{(-)}\right\rangle=e^{-i k_{+} R \varphi}|-\rangle,
\end{aligned}
$$

with normalized eigenspinors given by 23]

$$
|+\rangle=\frac{1}{\sqrt{1+\eta^{2}}}\left[\begin{array}{c}
i \eta \\
e^{-i \varphi}
\end{array}\right],|-\rangle=\frac{1}{\sqrt{1+\eta^{2}}}\left[\begin{array}{c}
1 \\
i \eta e^{-i \varphi}
\end{array}\right],
$$

where the SOI-dependent dimensionless $\eta$ parameter is given by

$$
\eta=\frac{2 k_{s o} R}{1+\sqrt{1+\left(2 k_{s o} R\right)^{2}}} .
$$


The corresponding spin-dependent wave numbers $k_{\sigma}$, with $\sigma= \pm$ satisfies the quadratic equation,

$$
\left(k_{F}^{2}-k_{\sigma}^{2}\right)\left[k_{F}^{2}-\left(k_{\sigma}-\frac{1}{R}\right)^{2}\right]-4 k_{s o}^{2}\left(k_{\sigma}-\frac{1}{2 R}\right)^{2}=0 .
$$

with $k_{\mathrm{F}}=\sqrt{2 m E_{\mathrm{F}} / \hbar^{2}}$ the Fermi wave number. For a sufficient large SOI and large ring radius to fulfill the adiabatic approximation $k_{ \pm} \gg 1 / R$, the spin-resolved wave numbers solutions of Eq.(14) are recasted as,

$$
k_{\sigma}=\sigma k_{s o}+\sqrt{k_{F}^{2}+k_{s o}^{2}} \simeq k_{F}+\sigma k_{s o} .
$$

the right hand side holds as long $k_{s o}^{2} \ll k_{F}^{2}$, which is true for typical 2DEG embedded in III-V semiconductor quantum wells.

Let us now consider a full cycle of the wave functions around the QR under a penetrating magnetic flux. Apart from the usual Aharonov-Bohm phase $\Phi_{A B}$ discussed above due to the presence of the SOI, a topological Berry phase $\theta_{B}^{ \pm}$have to be added to the spin-dependent wave functions. The Berry phase arises as a consequence of the phase factor acquired by the electron spin at the end of its loop around the QR as it adiabatically rotates (precesses) trying to follow the effective magnetic field $\boldsymbol{B}_{\text {eff }}$ due to SOI (see Fig, 2 , 2 $\mathrm{d}$ ). That is, $\left|\Psi_{k_{\sigma}}^{(+)}\right\rangle \rightarrow e^{i \Phi_{A B}} e^{-i \theta_{B}^{+}}\left|\Psi_{k_{\sigma}}^{(+)}\right\rangle$and $\left|\Psi_{k_{\sigma}}^{(-)}\right\rangle \rightarrow e^{i \Phi_{A B}} e^{-i \theta_{B}^{-}}\left|\Psi_{k_{\sigma}}^{(-)}\right\rangle$, and we have followed the sign convention in the Berry phase of Ref.21. The Berry phase is calculated as half of the material angle subtended by the effective magnetic field, such that the angle $\theta_{B}^{ \pm}$between the $z$-direction and the cone formed by the precessing spin satisfies

$$
\begin{aligned}
\cot \theta_{B}^{ \pm} & =\frac{\left\langle\Psi_{k_{\sigma}}^{( \pm)}\left|\sigma_{z}\right| \Psi_{k_{\sigma}}^{( \pm)}\right\rangle}{\sqrt{\left\langle\Psi_{k_{\sigma}}^{( \pm)}\left|\sigma_{x}\right| \Psi_{k_{\sigma}}^{( \pm)}\right\rangle^{2}+\left\langle\Psi_{k_{\sigma}}^{( \pm)}\left|\sigma_{y}\right| \Psi_{k_{\sigma}}^{( \pm)}\right\rangle^{2}}} \\
& = \pm \frac{\eta^{2}-1}{2 \eta}=\mp \frac{\hbar^{2}}{2 m \alpha_{s o} R}
\end{aligned}
$$

In the adiabatic approximation $k_{s o} R \gg 1$ (i.e. in the limit of vanishing spin-geometric phase [17, 19]) the parameter $\eta \rightarrow 1$ and the spin orientation of the electrons is described by the vector $\left\langle\Psi_{k_{\sigma}}^{(-)}|\boldsymbol{\sigma}| \Psi_{k_{\sigma}}^{(-)}\right\rangle=-\left\langle\Psi_{k_{\sigma}}^{(+)}|\boldsymbol{\sigma}| \Psi_{k_{\sigma}}^{(+)}\right\rangle=(\sin \varphi, \cos \varphi, 0)$. That is, the electron spin is oriented in-plane, radially toward or from the ring center of the QR, see Fig 2(b). Since the Rashba spin-orbit induced effective magnetic field is given by $\boldsymbol{B}_{\text {eff }} \sim \alpha_{\text {so }}(\boldsymbol{k} \times \boldsymbol{F})$, with $\boldsymbol{k}$ the wave vector of the moving electrons in an external electric field $\boldsymbol{F}$, then the spin-orientation is directed either, parallel or antiparallel to the effective magnetic field. For this case the overall Berry phase is just a constant and reduces to $\theta_{B}^{ \pm}= \pm \pi / 2$. 
Clearly these results above holds as long there are no scatterer points along the path of the electrons in the QR. The role of the QPCs on the scattering process, together with the phases involved are the focus of the following discussion.

\section{SCATTERING FORMALISM}

As depicted in Fig, 1 at the junction of each lead with the $\mathrm{QR}$ is a $\mathrm{QPC}_{i}$, where $i=1(2)$ refers to the left(right) quantum point contact. Additionally a third scatterer $\left(\mathrm{QPC}_{3}\right)$ is located at the upper arm of the QR. Now, it is important to mention that spin scattering mechanisms such as the spin-orbit mediated coupling with (piezoelectric) phonons, are believed to be the main source of spin-fliping in QRs as well as in quantum dots. However it is also well known theoretically and experimentally, that the associated relaxation times in these systems are extremelly large, ranging from milliseconds up to few seconds, see Refs.[37],[38] and [39]. This times are longer than the associated momentum and collision times in such ballistic systems and will not play a role here. Therefore any spin-flip processes at the QPCs will be subsequently neglected in this work. Also, without loss of generality, it will be assumed that the electron spin is preserved along the QR path. The spin states can be treated then as two independent channels of propagation. Consequently, for each spin channel we have three outgoing electronic waves with amplitudes $\left(\alpha_{i \sigma}^{\prime}, \beta_{i \sigma}^{\prime}, \gamma_{i \sigma}^{\prime}\right)$, and accordingly, three incoming waves with amplitudes $\left(\alpha_{i \sigma}, \beta_{i \sigma}, \gamma_{i \sigma}\right)$. Similarly, for the $\mathrm{QPC}_{3}$ we have two ingoing and two outgoing electronic waves with amplitudes $\left(\alpha_{3 \sigma}, \beta_{3 \sigma}\right)$ and $\left(\alpha_{3 \sigma}^{\prime}, \beta_{3 \sigma}^{\prime}\right)$, respectively (see Fig, 3 ).

It is worth recalling that in the adiabatic approximation, the presence of the Rashba-SOI in the QR do not actually intermixes the electron spins. The Rashba-SOI enters here solely a source of an AC phase of the traveling waves, as has been previously discussed. The AC phase adds up then to the $\mathrm{AB}$ and Berry phase acquired by the electrons waves in their round trip along the QR. We chose the convention that clockwise-oriented waves give rise to positive phases, whereas the waves moving in the opposite direction accumulates a negative phase.

Each lead-to-ring junction (the scattering of an electron with spin $\sigma$ at $\mathrm{QPC}_{1,2}$ ) is described by a $(3 \times 3)$ scattering matrix $\mathbb{S}$. Under the presence of a magnetic flux $\Phi$ penetrating the QR, time reversal invariance (TRI) is satisfied, leading to the known Onsager-Casimir 


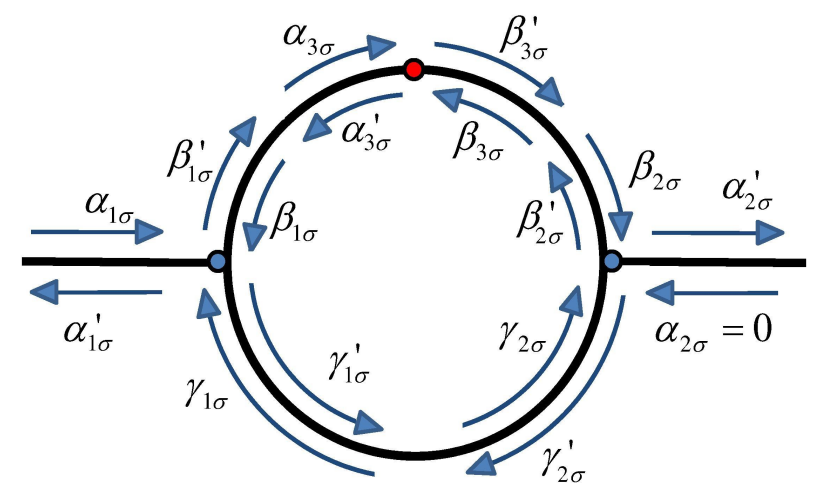

FIG. 3: (Color online) Diagram of the QR device attached to the leads. The arrows indicates the spin-resolved wave amplitudes during the spin-dependent scattering processes. The QPCs are indicated schematically by the small blue and red circles.

relations for the scattering matrix elements $S_{i j}(\Phi)=S_{j i}(-\Phi)$, where $i, j$ are the outgoing/ingoing channel, respectively. In the absence of the magnetic flux it reduces to the expected reciprocal relations (symmetricity of $\mathbb{S}$ ). This physically implies that the symmetric injection-ejection process of electrons at each junction (QPC) of the QR device is withhold. In addition, unitarity of $\mathbb{S}$ ensures current conservation at both, left and right QPCs. Therefore we can write

$$
\left[\begin{array}{c}
\alpha_{i \sigma}^{\prime} \\
\beta_{i \sigma}^{\prime} \\
\gamma_{i \sigma}^{\prime}
\end{array}\right]=\mathbb{S}\left[\begin{array}{c}
\alpha_{i \sigma} \\
\beta_{i \sigma} \\
\gamma_{i \sigma}
\end{array}\right], \quad \text { with } i=1,2
$$

as required for outgoing and ingoing waves amplitudes from standard scattering formalism. Given that flux conservation together with TRI, guarantee $\mathbb{S}^{-1}=\mathbb{S}^{*}$ which entails the symmetricity property $\mathbb{S}$, allow thus to propose

$$
\mathbb{S}=\left(\begin{array}{ccc}
R_{s}^{1 / 2} & \mathrm{a} T_{s}^{1 / 2} & \mathrm{a} T_{s}^{1 / 2} \\
\mathrm{a} T_{s}^{1 / 2} & \mathrm{~b} & \mathrm{c} \\
\mathrm{a} T_{s}^{1 / 2} & \mathrm{c} & \mathrm{b}
\end{array}\right),
$$

where we have defined $T_{s}$, as the electron transmission amplitude with spin $\sigma$ through a given QPC after formula (2), fulfilling $T_{s}(\varepsilon)+R_{s}(\varepsilon)=1$. The parameters $\mathrm{a}$, $\mathrm{b}$ and $\mathrm{c}$ are real coefficients to be determined. In (18) the diagonal matrix elements, $S_{i i}$, represent the 
reflection amplitude of the $i-t h$ channel, whereas the off-diagonal elements $S_{i j}(i \neq j)$ characterizes the transmission amplitudes from channel $i$ to $j$ at a given junction. By demanding the unitary condition of the incident flux, i.e. $\mathbb{S}^{\dagger} \cdot \mathbb{S}=\boldsymbol{I}_{3}$, with $\boldsymbol{I}_{N}$ the $(N \times N)$ identity matrix, the parameters a, b and $\mathrm{c}$ in 18 are readily obtained, yielding

$$
\mathrm{a}=\frac{1}{\sqrt{2}} ; \mathrm{b}=\frac{-R_{s}^{1 / 2} \pm 1}{2} ; \text { and } \mathrm{c}=\frac{-R_{s}^{1 / 2} \mp 1}{2}
$$

notice that $b$ and $c$ depend explicitly on the effective energy $\varepsilon$ through the reflection probability of each QPC.

Let us consider now the incident and reflected electronic waves at one fourth cycle in the upper branch of the $\mathrm{QR}$, just at the $\mathrm{QPC}_{3}$ location (see Fig. 3). In such a case, by following usual rules of transfer matrix formalism, 25] the amplitudes of the wave functions can be related to those of the $\mathrm{QPC}_{1}$ as follows

$$
\left[\begin{array}{c}
\alpha_{3 \sigma} \\
\alpha_{3 \sigma}^{\prime}
\end{array}\right]=\left(\begin{array}{cc}
\exp \left(i \varphi_{2 \sigma}\right) & 0 \\
0 & \exp \left(-i \varphi_{1 \sigma}\right)
\end{array}\right)\left[\begin{array}{c}
\beta_{1 \sigma}^{\prime} \\
\beta_{1 \sigma}
\end{array}\right],
$$

and similarly with respect to the wave amplitudes at $\mathrm{QPC}_{2}$,

$$
\left[\begin{array}{c}
\beta_{2 \sigma} \\
\beta_{2 \sigma}^{\prime}
\end{array}\right]=\left(\begin{array}{cc}
\exp \left(i \varphi_{2 \sigma}\right) & 0 \\
0 & \exp \left(-i \varphi_{1 \sigma}\right)
\end{array}\right)\left[\begin{array}{c}
\beta_{3 \sigma}^{\prime} \\
\beta_{3 \sigma}
\end{array}\right],
$$

where $\varphi_{1}$ is the net phase acquired by an electron traversing half of the upper arm semicircle in the clockwise direction, whiles $\varphi_{2}$ corresponds to the phase acquired by the electron in the same segment but in the counterclockwise direction. For spin up/down $(\sigma= \pm)$ electrons such phases read explicitly

$$
\begin{gathered}
\varphi_{1 \pm}=\frac{\pi}{2}\left(k_{ \pm} R-\phi+\frac{1}{2}\right), \\
\varphi_{2 \pm}=\frac{\pi}{2}\left(-k_{\mp} R-\phi+\frac{1}{2}\right),
\end{gathered}
$$

with $k_{\sigma}$ as given by of Eq. (15) and $\phi=\Phi / \Phi_{o}$ is the magnetic flux in units of the magnetic flux quanta $\left(\Phi_{o}=h / e\right)$. The added quantity $\pi / 4$ comes from the accumulated Berry phase in the semicircle. Note that due to the Rashba-SOI, Kramers degeneracy is broken, and hence an electron -at a given Fermi energy-, with spin-up orientation $(\sigma=+)$ and traveling with a wavenumber $k_{+}$will be elastically backscattered with a wavenumber $-k_{-}$, as given by Eq. (15). Similarly occurs for the opposite spin case $(\sigma=-)$. 
For the lower arm of the QR the wave amplitudes are transferred according to

$$
\left[\begin{array}{c}
\gamma_{1 \sigma} \\
\gamma_{1 \sigma}^{\prime}
\end{array}\right]=\left(\begin{array}{cc}
\exp \left(i \varphi_{4 \sigma}\right) & 0 \\
0 & \exp \left(-i \varphi_{3 \sigma}\right)
\end{array}\right) \mathbb{T}_{l}\left[\begin{array}{c}
\gamma_{2 \sigma}^{\prime} \\
\gamma_{2 \sigma}
\end{array}\right]
$$

with $\varphi_{3 \sigma}=2 \varphi_{1 \sigma}$ and $\varphi_{4 \sigma}=2 \varphi_{2 \sigma}$. In equation (24) we have included for completeness the presence of a fourth scatterer $\left(\mathrm{QPC}_{4}\right)$ at the lower arm of the $\mathrm{QR}$, characterized by the transfer matrix $\mathbb{T}_{l}$. For the case in which a constriction-free path in the lower branch of the QR is considered, then we use $\mathbb{T}_{l}=\boldsymbol{I}_{2}$.

After some standard scattering theory operations within the transfer matrix approach, together with TRI symmetry properties lead us to express

$$
\left[\begin{array}{c}
\beta_{3 \sigma}^{\prime} \\
\beta_{3 \sigma}
\end{array}\right]=\mathbb{T}_{u}\left[\begin{array}{c}
\alpha_{3 \sigma} \\
\alpha_{3 \sigma}^{\prime}
\end{array}\right]
$$

in which the transfer matrix $\mathbb{T}_{u}$ has the form

$$
\mathbb{T}_{u}=\left(\begin{array}{cc}
\frac{1}{t_{s}} & -\frac{r_{s}}{t_{s}} \\
-\frac{r_{s}^{*}}{t_{s}^{*}} & \frac{1}{t_{s}^{*}}
\end{array}\right)
$$

and we will assume that $t_{s}(\varepsilon)=T_{s}(\varepsilon)^{1 / 2}$ and $r_{s}(\varepsilon)=R_{s}(\varepsilon)^{1 / 2}$. They stand for the transmission and reflection amplitudes of the $\mathrm{QPC}_{3}$, respectively. On the other hand, it is convenient to define the diagonal matrix

$$
\boldsymbol{\Phi}_{u, l}^{\sigma}=\left(\begin{array}{cc}
\exp \left(i \varphi_{2 \sigma, 4 \sigma}\right) & 0 \\
0 & \exp \left(-i \varphi_{1 \sigma, 3 \sigma}\right)
\end{array}\right)
$$

where the subscripts $(u, l)$ stands for (upper, lower) part of the QR, respectively. After some algebraic manipulations (see appendix A), a closed expression is obtained for the total transmitted amplitude $\alpha_{2 \sigma}^{\prime}$ of the outgoing electrons at the right lead of the QR, yielding

$$
\left[\begin{array}{c}
\alpha_{2 \sigma}^{\prime} \\
0
\end{array}\right]=\frac{2 T_{s}}{\left(R_{s}^{1 / 2}+\lambda\right)^{2}} \boldsymbol{\lambda}\left(\mathbb{P}^{\sigma}\right)^{-1} \boldsymbol{\Phi}_{u}^{\sigma} \mathbb{T}_{u} \boldsymbol{\Phi}_{u}^{\sigma}\left[\begin{array}{c}
-\lambda \\
1
\end{array}\right]
$$

where $\mathbb{P}^{\sigma}$ is a $2 \times 2$ matrix depending upon the QR parameters (Appendix A) while the matrix $\boldsymbol{\lambda}$ is given by

$$
\boldsymbol{\lambda}=\left(\begin{array}{cc}
\lambda & 1 \\
0 & 0
\end{array}\right) ; \lambda= \pm 1
$$


It is worth to emphasize that Eq.(28) summarizes one of the main results of this work. Its importance relies on the fact that, all the relevant physical information of the coherent transport phenomena, such as the electron scattering, the change of phase (including AB, $\mathrm{AC}$ and Berry phases) as well as the interference phenomenon occurring in the QR setup, are all comprised in $\alpha_{2 \sigma}^{\prime}$. The two-probe Landauer conductance, 32] of the QR is described by

$$
G(\varepsilon)=\sum_{\sigma} G_{\sigma}(\varepsilon)=\frac{e^{2}}{h} \sum_{\sigma}\left|\alpha_{2 \sigma}^{\prime}(\varepsilon)\right|^{2},
$$

then it is possible to find a closed analylical formula for the spin-resolved conductance of the $\mathrm{QR}$ device, and it is given by

$$
G_{\sigma}(\varepsilon)=\frac{e^{2}}{h} \frac{4 T_{o}^{2}(\varepsilon)\left[1+T_{s}(\varepsilon)+2 \sqrt{T_{s}(\varepsilon)} \cos \left(2 \varphi_{\sigma}\right)\right] \sin ^{2} \theta}{\left[A_{-}(\varepsilon)-A_{o}(\varepsilon) \cos (2 \theta)+A_{+}(\varepsilon) \sqrt{T_{s}(\varepsilon)} \cos \left(2 \varphi_{\sigma}\right)\right]^{2}+4 T_{o}^{2}(\varepsilon) \sin ^{2}(2 \theta)},
$$

where we have defined $A_{ \pm}(\varepsilon)=2-T_{o}(\varepsilon) \pm 2 \sqrt{1-T_{o}(\varepsilon)}$ and $A_{o}(\varepsilon)=A_{+}(\varepsilon)+A_{-}(\varepsilon)$. The angular parameters describing the different phases are namely, the dynamical phase $\theta=\pi k_{\mathrm{F}} R$, and aspin-dependent phase

$$
\varphi_{\sigma}=\pi\left(\frac{1}{2}-\phi+\sigma \phi_{s o}\right)
$$

that contain all the involved phases, with $\phi$ and $\phi_{s o}=k_{s o} R$ being the $\mathrm{AB}$ and the $\mathrm{AC}$ phases, the $\pi / 2$ comes from the Berry phase. The transmission coefficient is the same in both $\mathrm{QPC}_{1,2}, T_{o}(\varepsilon)$, as they are supposed to be identical by construction, while the same quantity at $\mathrm{QPC}_{3}$ is denoted by $T_{s}(\varepsilon)$. We emphasize though that the formalism is quite general and admits different transmission probabilities of $\mathrm{QPC}_{1}$ and $\mathrm{QPC}_{2}$. Here for the sake of clearity, we have considered only the case in which both QPCs are identical. This allow us to concentrate on the interplay of other important parameters such varying the aperture of $\mathrm{QPC}_{3}$ whiles the magnetic field and/or the Rashba spin-orbit coupling are tuned. As shown above by considering symmetric QPCs leads us to arrive to a relatively simple and explicit formula for the spin conductance of the device (Eq. 31) which in turn enable us to a deeper understanding of the resonance and anti-resonance behavior of the QR device, discussed in further detail below.

Note that the conductance result of Eq. (31) hold in general, that is, independent of the transmission probability model $T_{s}$ considered for the QPCs. Here we will use the saddle 
point potential profile of Fertig and Halpering discussed in Sec.II to model the transmission probability of the QPCs, explicitly Eq.(2) and Eq.(3). Due to its strong dependence on the magnetic flux (magnetic field), as well as with the lateral and transversal electrostatic confinement at the constrictions, the QR device proposed offers additional degrees of freedom to manipulate spin-transport and interference phenomena. Indeed, as predicted by Eq.(31), the particular geometry choice of the QR device with tunable QPCs, results in a conductance which exhibits strong oscillations with the strength of the magnetic flux and Rashba-SOI, oscillations which in turn are modulated in a non-trivial way by the opacity of the QPCs, as it will be discussed later in more detail.

The cases with different confinement strengths for $\mathrm{QPC}_{1}$ and $\mathrm{QPC}_{2}$, together with the one of a fourth $\mathrm{QPC}_{4}$ at the lower arm of the $\mathrm{QR}$ are beyond the scope of the present work and will be treated in a subsequent publication. We anticipate though that due the cumbersome expressions in such cases, it would render quite difficult to arrive to close analytical formulas of the conductance of the QR device for such cases.

\section{A. Spin resolved conductance: limit cases}

It is illustrative to analyze the spin resolved conductance at some limit cases. We can distinguish three extreme scenarios for the conductance behavior depending upon the opacity or the aperture of the QPCs. Namely, case $(i)$, maximum transparency at each $\mathrm{QPC}_{i}$, case (ii), maximum transparency only at $\mathrm{QPC}_{3}$, and case $\left(\right.$ iii) maximum transparency at $\mathrm{QPC}_{1}$ and $\mathrm{QPC}_{2}$ whereas $\mathrm{QPC}_{3}$ exhibits a variable transparency.

For case (i) we have $T_{o}(\varepsilon)=T_{s}(\varepsilon)=1$, thus $A_{ \pm}=1, A_{o}=2$, and the spin-resolved (31) conductance is readily compacted to

$$
G_{\sigma}=\frac{e^{2}}{h}\left[\frac{16 \cos ^{2} \varphi_{\sigma} \sin ^{2} \theta}{\left(1-2 \cos (2 \theta)+\cos \left(2 \varphi_{\sigma}\right)\right)^{2}+4 \sin ^{2}(2 \theta)}\right],
$$

which is only a function of the dynamic phase $\theta$, and the round trip phase $\varphi_{\sigma}$ due to the accumulated AB, AC and Berry phases. The expression between large square brackets, is just the spin-dependent transmission probability of the QR. In the absence of Rashba-SOI and neglecting the Berry phase (i.e. with $\varphi_{\sigma}=-\pi \phi$ ) it reduces to the formula derived by Vasilopoulus et al. 27] for the transmission probability in an AB-QR without scatterers [Eq.(7) in Ref 27]. Clearly formula (33) leads to resonances and antiresonances in the total 
conductance. The condition for the appearance of resonances with a maximum in the spinconductance $\left(G_{\sigma}=1\right)$ are hold whenever $\varphi_{\sigma}=n \pi$, with $n$ integer. On the other hand, all the antiresonances (zeros of the spin-conductance) appear when $\varphi_{\sigma}=\frac{\pi}{2} n$. By fixing the

magnetic flux $\phi=\frac{1}{2}$ then these maximum resonances/anti resonances are present as long $\phi_{\mathrm{SO}}^{\max }=n$ and $\phi_{\mathrm{SO}}^{\min }=\frac{n}{2}$, with $n$ an integer and odd number, respectively.

Case (ii): perfect transparency at $\mathrm{QPC}_{3}$ only, i.e. backscattering-free regime in $\mathrm{QPC}_{3}$, which entails $T_{s}(\varepsilon)=1$ together with $T_{o}(\varepsilon) \neq 1$ in the other two QPCs. Considering these requirements the condition for maximum conductance is dictated by

$$
\cos \left(2 \phi_{\sigma}\right)=\frac{T_{o}(\varepsilon)^{2}+4\left[2\left(1+\sqrt{R_{o}(\varepsilon)}\right)-\left(2+\sqrt{R_{o}(\varepsilon)}\right) T_{o}(\varepsilon)\right] \cos (2 \theta)}{\left(T_{o}(\varepsilon)-2-2 \sqrt{R_{o}(\varepsilon)}\right)^{2}} .
$$

Case (iii): perfect transparency at $\mathrm{QPC}_{1}$ and $\mathrm{QPC}_{2}\left(T_{o}(\varepsilon)=1\right)$ whereas $T_{s}(\varepsilon) \neq 1$ in $\mathrm{QPC}_{3}$. Here the maximum spin-conductance is expected as long that

$$
\cos \left(2 \phi_{\sigma}\right)=\frac{1 \pm \sqrt{R_{s}(\varepsilon)} \sin \theta}{\sqrt{T_{s}(\varepsilon)}}
$$

These expressions above will be useful in the understanding of the origin of the QRconductance oscillations and will be discussed in more detail in the next section. Finally, we should mention that there is yet another possible configuration. This is such that $\mathrm{QPC}_{3}$ is totally opaque (closed) with $R_{s}(\varepsilon)=1$ (i.e. $T_{s}(\varepsilon)=0$ ) and finite transmitivity at the other two QPCs. From Eq. 31) we can infer that the AB and AC oscillations are totally absent in this situation, as they should, due the truncated path for the moving electrons in the upper arm of the QR because of the suppressed quantum interference.

\section{NUMERICAL RESULTS AND DISCUSSION}

Unless otherwise specified, the numerical results reported here were calculated using a QR radius of $R=250 \mathrm{~nm}$ defined on an InAs-based heterostructure with electron effective mass of $m=0.023$. Hereafter, the two-probe Landauer conductance $G(\varepsilon)$ (30) will be referred as $G$, whiles for simplicity the characteristic longitudinal and transversal energy confinements at the lead-to-ring junction will be assumed to be equal $\left(\hbar \omega_{x}=\hbar \omega_{y}\right)$, and from now on denoted by $\hbar \omega_{j}$. Notice that even large changes between them will not change the main physical results as the transmission probabilities defined in Eq.(2) will follow essentially the 

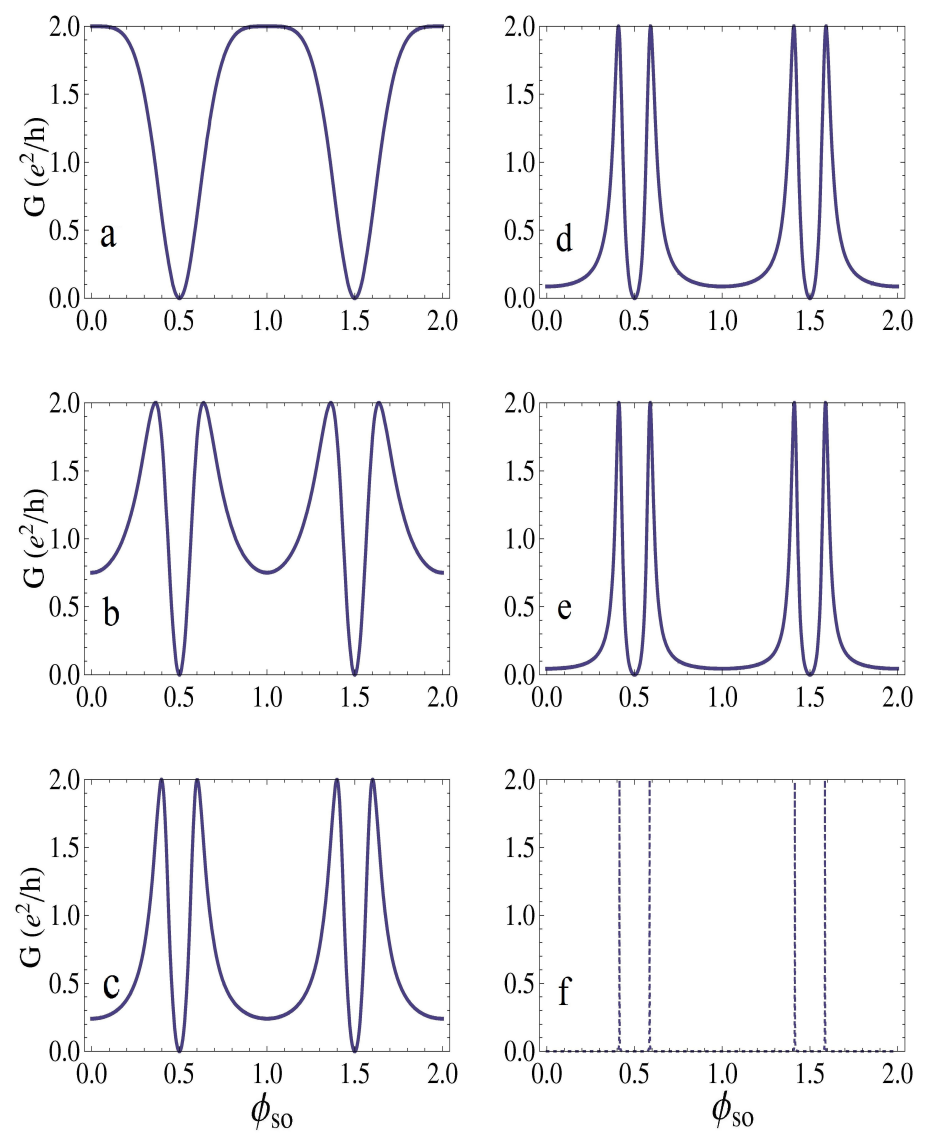

FIG. 4: Evolution of the conductance quantum oscillations as a function of the AC phase $\phi_{\text {SO }}$. We have fixed $T_{s}(\varepsilon)=1$ and the AB-phase to $\phi=0.5$, the latter corresponds to a field of $B=10$ $\mathrm{mT}$ for the QR structure considered here. The transmission coefficients used at the lead-to-ring junctions, in the panels $a, b, c, d, e$ and $f$; correspond to $T_{o}(\varepsilon)=1,3 / 4,1 / 2,1 / 3,1 / 4$ and $10^{-3}$, respectively, or equivalently, to confinement energies strenghts of $\hbar \omega_{j}=0.2,2.44,2.72,2.94,3.08$ and $10.2 \mathrm{meV}$.

same step-like trend in such cases. [33] The typical values for $\hbar \omega_{j}$ are in the range of $0.8 \sim 3$ meV [34, 35, whereas the experimental values of the Rashba parameter $\alpha_{\text {so }}$ are between $20 \sim 40 \mathrm{meV} n \mathrm{~m}$ (in InAs-based 2DEGs [36, 37]) and will correspond to AC-phases $\phi_{\mathrm{so}}$ of about 0.47 and 0.95 , respectively.

Figure 4 displays the periodic quantum oscillations of $G(31)$, versus the $\mathrm{AC}$ phase $\phi_{\text {so }}$ in the interval $[0,2]$ for different values of transmission coefficients at the lead-to-ring junctions $T_{o}(\varepsilon)$, together with $\mathrm{QPC}_{3}$ fully open. The latter implies $T_{s}(\varepsilon)=1$, which entails $\hbar \omega_{j}=0.2 \mathrm{meV}$ and $\phi=0.5$ for the AB-phase. Moreover the dynamic phase has been set to 
$\theta=\pi k_{\mathrm{F}} R=39$, with $k_{\mathrm{F}}=\sqrt{2 \pi n_{e}}$ and $n_{e}=3.93 \times 10^{10} \mathrm{~cm}^{-2}$. The panel of Fig.4. $a$ correspond to a situation in which both $\mathrm{QPC}_{1,2}$ are fully open $\left(T_{o}=1\right)$, so there is no scattering of the moving electrons around the QR. The oscillations of the conductance with $\phi_{\text {so }}$ are a clear manifestation of the spin-interference. As described in Case (i) above, the maximums (resonances) of the conductance are present always that $\phi_{\mathrm{SO}}^{\max }=n$, here with $n=0,1,2$ whereas the minimum (anti-resonances) appear as long $\phi_{\mathrm{SO}}^{\min }=\frac{n}{2}$, with $n=1$, 3 . As soon $\mathrm{QPC}_{1,2}$ are set to partial transparency $\left(T_{o} \neq 1\right)$, the pattern of the quantum oscillations of the conductance changes drastically and the resonances follows Eq.(34) producing double peaks around each anti-resonance which now appears at $\phi_{\mathrm{SO}}^{\min }=\frac{n}{2}$, with $n$-integer.

To further analyze the results let us consider the particular case of Fig.4c in which both $\mathrm{QPC}_{1}$ and $\mathrm{QPC}_{2}$ are set to half transparency $\left(T_{o}(\varepsilon)=R_{o}(\varepsilon)=1 / 2\right)$ while $\mathrm{QPC}_{3}$ is totally open $\left(T_{s}=1\right)$. From Eq. (34) the resonances of the conductance are governed by

$$
\phi_{\mathrm{SO}, \pm}^{\max }=n \pm \frac{1}{2 \pi}\left[c_{1}+c_{2} \operatorname{arcCos}(\cos 2 \theta)\right],
$$

with $n$ integer, $c_{1}=17-12 \sqrt{2}$ and $c_{2}=1-c_{1}$. On the other hand the the minimums of the conductance arises here as long $\phi_{\mathrm{SO}}^{\min }=\frac{n}{2}$, with $n$-integer (see Table I). Clearly as we would expect, the width of the resonances diminishes as the opacity of the $\mathrm{QPC}_{1,2}$ increases. In the limit of vanishing transparency, when $T_{o}(\varepsilon)=0.001$ (see Fig, 4 ), each QPC at the lead-toring junctions have thus the largest opacity. In this scenario a low-probability flux through the lead-to-ring junctions is allowed -despite the strong confinement potential imposed-given rise to the sharp resonances observed as the eigenenergies of the $\mathrm{QR}$ are being mapped.

In Figure 5. a(b) we show plots of the quantum conductance of the $\mathrm{QR}$ with $\mathrm{QPC}_{3}$ fully transparent $\left(T_{s}=1\right)$ and the other $\mathrm{QPC}_{1,2}$ partially open, as a function of $\mathrm{AB}$-phase $\phi$ and confinement strength $\hbar \omega_{j}$, in the range between $[-1,1]$ and $[0,6] \mathrm{meV}$, respectively. The plot in panels (a) and (b) show $G$ for two values of AC-phase ( $\phi_{\mathrm{SO}}=0.5,1.0$, respectively). Notice the shifting of the $G$-oscillation pattern as a function of $\phi$ by tuning selectively two values for the SOI parameter $\alpha_{\mathrm{SO}}$. The behavior of the conductance with $\hbar \omega_{j}$ exhibits a clear crossover around $\hbar \omega_{j}=2.7 \mathrm{meV}$ regardless of the AB-phase value. Indeed, for $\hbar \omega_{j}<2.72 \mathrm{meV}$ (equivalent to $T_{j}=0.5$ ) the conductance versus $\phi$ shows the expected widespread periodic AB-oscillations [23, 25] with maximum amplitude $2 e^{2} / h$. However for confinement strengths $\hbar \omega_{j}>2.72 \mathrm{meV}$ the quantum transport through the QR device is strongly influenced by the opacity of $\mathrm{QPC}_{1,2}$ (larger coupling lead-to-ring) and rapidly reduces to vanishing values. 
As a consequence the widespread periodic oscillations of $G$ evolves into very sharp resonant features. This behavior can be seen more clearly in panel 5.d showing a density map of $G$ against the plane $\left(\phi, \hbar \omega_{j}\right)$. The white dotted vertical line at the transition regime $\hbar \omega_{j}=2.72$ $\mathrm{meV}$ is drawn here just to guide the eye.

It is illustrative to have a close look to the model transmission coefficient (2) for the QPCs, as a function of $\hbar \omega_{j}$ and the dynamic phase $\theta$. This is the aim of the Fig.5. (e). The transmission probability $T_{\mathrm{SD}}$, is adiabatically modulated following a smooth step-like profile from its maximum value $\left(T_{\mathrm{SD}}=1\right)$ at its plateau, to essentially zero at relatively large values of $\hbar \omega_{j}$ and $\theta$. As a result the coherent quantum interference phenomena and the electronic flux in the QR device is affected accordingly as $T_{\mathrm{SD}}$ in $\mathrm{QPC}_{1,2}$ is modulated. For instance, the transmission probability at the neighborhood of $\theta_{\mathrm{F}} \approx 40$ (i.e. $E_{\mathrm{F}} \approx 43.2$ $\mathrm{meV}$ ), indicated with a solid black (white dashed) line in Fig.5 $5(\mathrm{e})$, changes significantly as $\hbar \omega_{j}$ is varied in the interval between 2.4 and $3.6 \mathrm{meV}$. Outside this range, the values of $T_{\mathrm{SD}}$ are basically uniform; being maximal for $\hbar \omega_{j}<2.4 \mathrm{meV}\left(T_{\mathrm{SD}} \rightarrow 1\right)$, and minimal $\left(T_{\mathrm{SD}} \rightarrow 0\right)$ when $\hbar \omega_{j}>3.6 \mathrm{meV}$ (see Fig/5). Such behavior of $T_{\mathrm{SD}}$ at each lead-to-ring junction $\left(\mathrm{QPC}_{1,2}\right)$ explains well the two drastic different features of the $G$-oscillations with respect to $\hbar \omega_{j}$; from the widespread of the periodic $G$-oscillation, to the resonant response, as shown in Fig $55 a(b)(c)$.

We now turn to another interesting phenomenology. This concerns the situation in which the $G$-oscillation pattern (as a function of $\phi$ ) are shifted uniformly just by tuning selectively the Rashba SOI parameter $\alpha_{\mathrm{SO}}$. These are represented in Fig. $5 a$ as the two superimpose conductance plots corresponding to the two different values for the AC-phase, $\phi_{\mathrm{so}}=0.5(1.0)$. From Eqs. (31) and (32) it is easy to show that maximums in $G$ should appear as long $\phi_{ \pm}^{\max }=\frac{1}{2} \mp \phi_{\mathrm{SO}}-n$, with $n$ integer. Therefore the dashed horizontal lines in panel $(b)$ at $\phi= \pm 0.5$ values, would correspond to the conductance maximums [dark curve in panel $(a)$ ] for which $\phi_{\mathrm{so}}=1.0$, while $G$-oscillations of the $\phi_{\mathrm{so}}=0.5$ [lighter curve in panel $(a)$ ] case appears shifted by a phase of 0.5 as we would expect.

In Figures 6 $a(b)$ we present the plots of the evolution of the quantum conductance oscillations of the QR-device as a function of the AC-phase $\phi_{\text {so }}$ and the dynamic phase $\theta$, for a fix AB-phase of $\phi=0.5$ and confinement strength of $\hbar \omega_{j}=0.2 \mathrm{meV}$ (which imposes $T_{o}=1$ for $\left.\mathrm{QPC}_{1,2}\right)$, whiles setting $\mathrm{QPC}_{3}$ at its maximum transparency $\left(T_{s}=1\right)$. The periodic oscillatory patterns found for $G$ against $\phi_{\mathrm{so}}$ respond the to $\mathrm{AC}$ spin-interference 

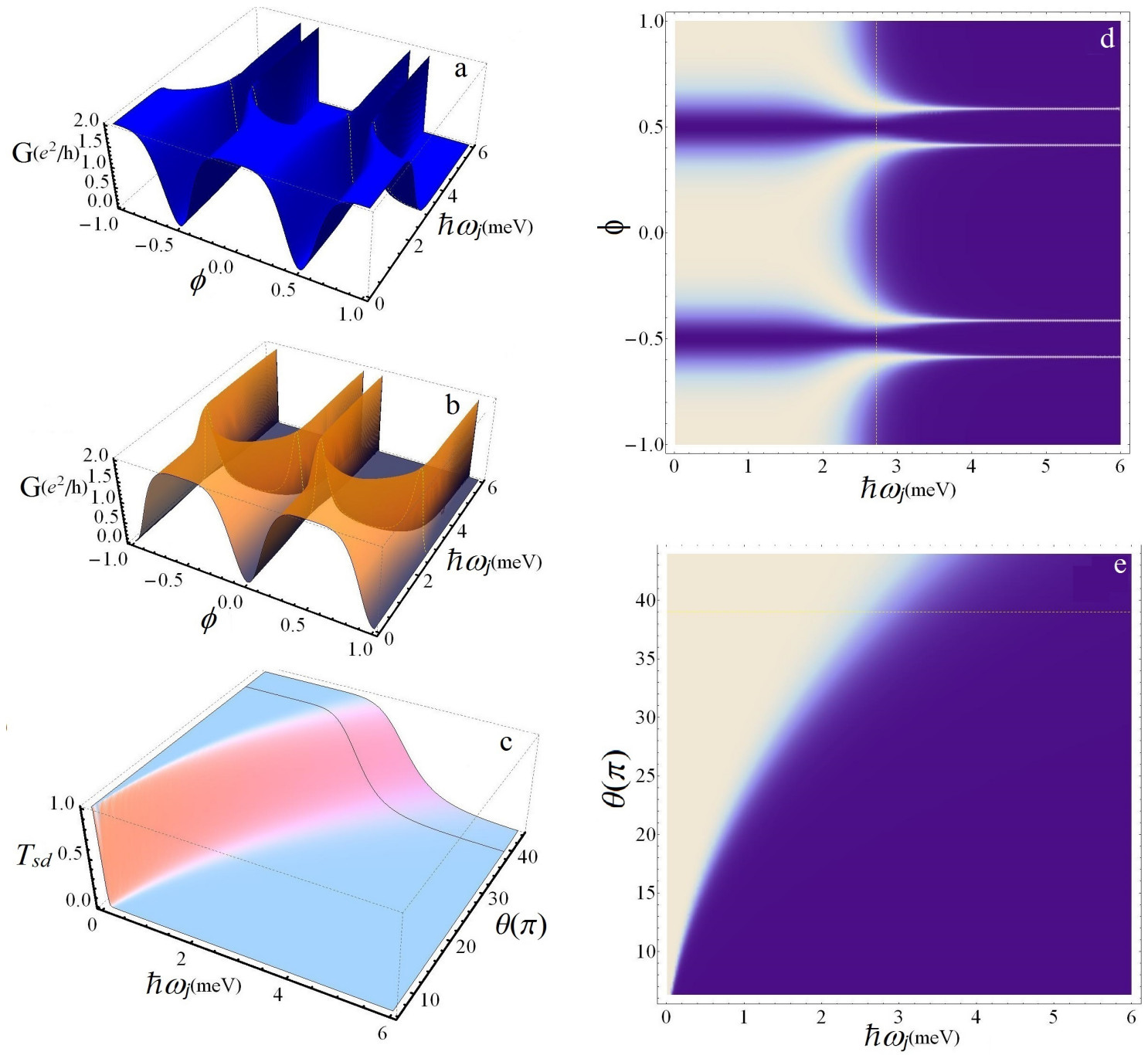

FIG. 5: (Color online) Panels (a),(b) and (d) show the oscillations of $G$ as a function of the AB phase $\phi$ and confinement energy $\hbar \omega_{j}$. For plot in panels (a) and $(\mathrm{d})$, we use $\phi_{\text {so }}=0.5\left(\alpha_{\text {so }}=21\right.$ $\mathrm{meV} \mathrm{nm})$, while in panel (b) we have set $\phi_{s o}=1.0\left(\alpha_{s o}=42 \mathrm{meV} \mathrm{nm}\right)$. The dotted vertical line in panel (d), points the value $\hbar \omega_{j}=2.72 \mathrm{meV}$ (equivalent to $T_{j}=1 / 2$ ). The panels (c) and (e) present the oscillations of transmission coefficient (2), as a function of $\hbar \omega_{j}$ at the junctions, and the phase $\theta$. The limit of the Fermi dynamic phase $\theta_{\mathrm{F}} \approx 39.2$ (solid black/dashed white line) is indicated in panel (c)/(e). The light/dark distribution of the density maps corresponds to the higher/smaller values of $G$, respectively.

effects induced by the SOI-R, as had been earlier predicted and measured for biased ACQRs at low temperature.[20, 31, 37, 38] On the other hand, the periodicity of $G$ against the dynamic phase $\theta$ comes from the $\sin ^{2} \theta$ dependence of the spin-resolved conductance in 

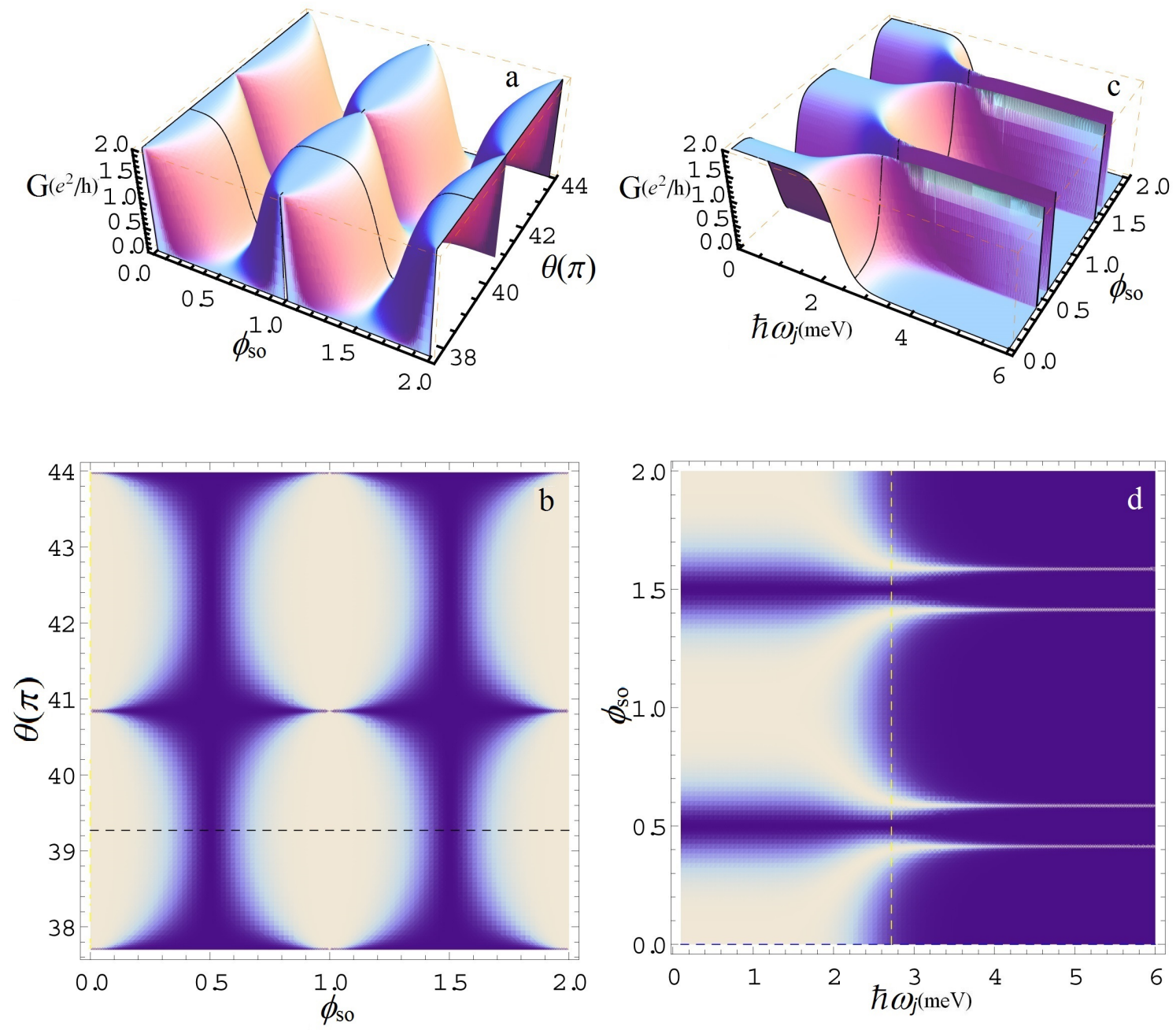

FIG. 6: (Color online) Panels $a$ ) and (b) shows $G$-oscillations, as a function of the AC phase $\phi_{\mathrm{SO}}$ and the dynamic phase $\theta$. The limit of the Fermi dynamic phase $\theta_{\mathrm{F}} \approx 39.2$ (dashed dark line), is indicated. The panel $(c) /(d)$ presents the same, as a function of the confinement $\hbar \omega_{j}$ at the junctions, and the phase $\phi_{\mathrm{SO}}$ in a density map for $G$. The limit of $\hbar \omega_{j}=2.72 \mathrm{meV}$ (dashed light line) is indicated. The light-dark distribution within the density map corresponds to a strong-weak gradient for the values of $G$. All plots were calculated at a magnetic field $B=10 \mathrm{mT}$, corresponding to a AB-phase of $\phi=0.5$ for the studied QR.

Eq.(31) and arises due the coherent quantum interference of the winding electrons around the QR.

The oscillatory pattern changes drastically when a variable lateral confinement strength of $\mathrm{QPC}_{1,2}$ is considered while maintaining $\mathrm{QPC}_{3}$ fully open. This is shown in Fig, $6 . c(d)$ 
TABLE I: Extremes of (34) obtained from Eq.(36) for a few values of $n$. We have fixed $T_{o}=0.5$, and $\theta=39$.

\begin{tabular}{cccr}
\hline \hline$n$ & $\phi_{\mathrm{SO} \pm}^{\min }$ & $\phi_{\mathrm{SO}-}^{\max }$ & $\phi_{\mathrm{SO}+}^{\max }$ \\
\hline \hline-2 & -1 & -2.39841 & -1.60159 \\
-1 & -0.5 & -1.39841 & -0.60158 \\
0 & 0 & -0.39841 & 0.39841 \\
1 & 0.5 & 0.60158 & 1.39841 \\
2 & 1 & 1.60159 & 2.39841 \\
\hline \hline
\end{tabular}

for a fix dynamic phase of $\theta=39$. Similar as it occurs in Fig. $5 c(d)$ the evolution of the quantum oscillations of $G$ against the AC-phase reaches a crossover into a well-defined abrupt resonances as the QPC confinement energy $\hbar \omega_{j}$ is increased. The overall phenomenology shown here is identical of what arises in Fig. $5 c(d)$ by just replacing $\phi \leftrightarrows \pm \phi_{\mathrm{SO}}$. This is expected by the symmetry of the $\mathrm{AC}$ and $\mathrm{AB}$ phases as can be verified from Eq.(32).

The influence of the transmitivity of $\mathrm{QPC}_{3}$ in the conductance of the QR device assuming identical transmitivity to that of the other two QPCs at the lead-to-ring junctions is investigated in Fig.7. The conductance $G$ is calculated as a function of phases $\phi$ and $\phi_{\mathrm{So}}$ for four different values of the transmission probability of the $\mathrm{QPC}_{1,2,3}\left(T_{o}=T_{s}=1,3 / 4,1 / 2\right.$, and 1/3) and plotted in Fig.7, $a-c$, respectively. In Fig.7 $a$ the well known oscillating periodic response of $G$ for a QR without scatterers (i.e. in the absence of QPCs) are nicely reproduced here. For instance, similar behaviors had been reported for an AB ring without any scatter in the arms of the QR. [38] The periodic plateau formation at $2 e^{2} / h$ with the $\mathrm{AB}$ and AC phases in Fig.7 a are also in agreement with earlier results for InGaAs/InAlAs QR structures. 23. The new behavior in the QR device arises here as soon a finite scattering of the traveling electrons is considered due the presence of partially open QPCs at the lead-to-ring junctions and in the upper arm of the $\mathrm{QR}$. As a consequence of effectively closing the $\mathrm{QPC}_{1,2,3}$ the observed plateaus of $G$ (in units $2 e^{2} / h$ ) shown in Fig.7 $7 a$ at multiple half-integer values of $\phi$ and $\phi_{\mathrm{SO}}$, evolves adiabatically to depression-like structures, forming periodic crater-type features instead, see Fig. 7b-c. Due the reduced flux of electrons allowed to enter the QR by diminishing $T_{o}$ and $T_{s}$, a rather sensitive and strong tendency to fade away the amplitude of the overall conductance response is predicted, as being shown in panel $d$ Fig.7. Notice that, 


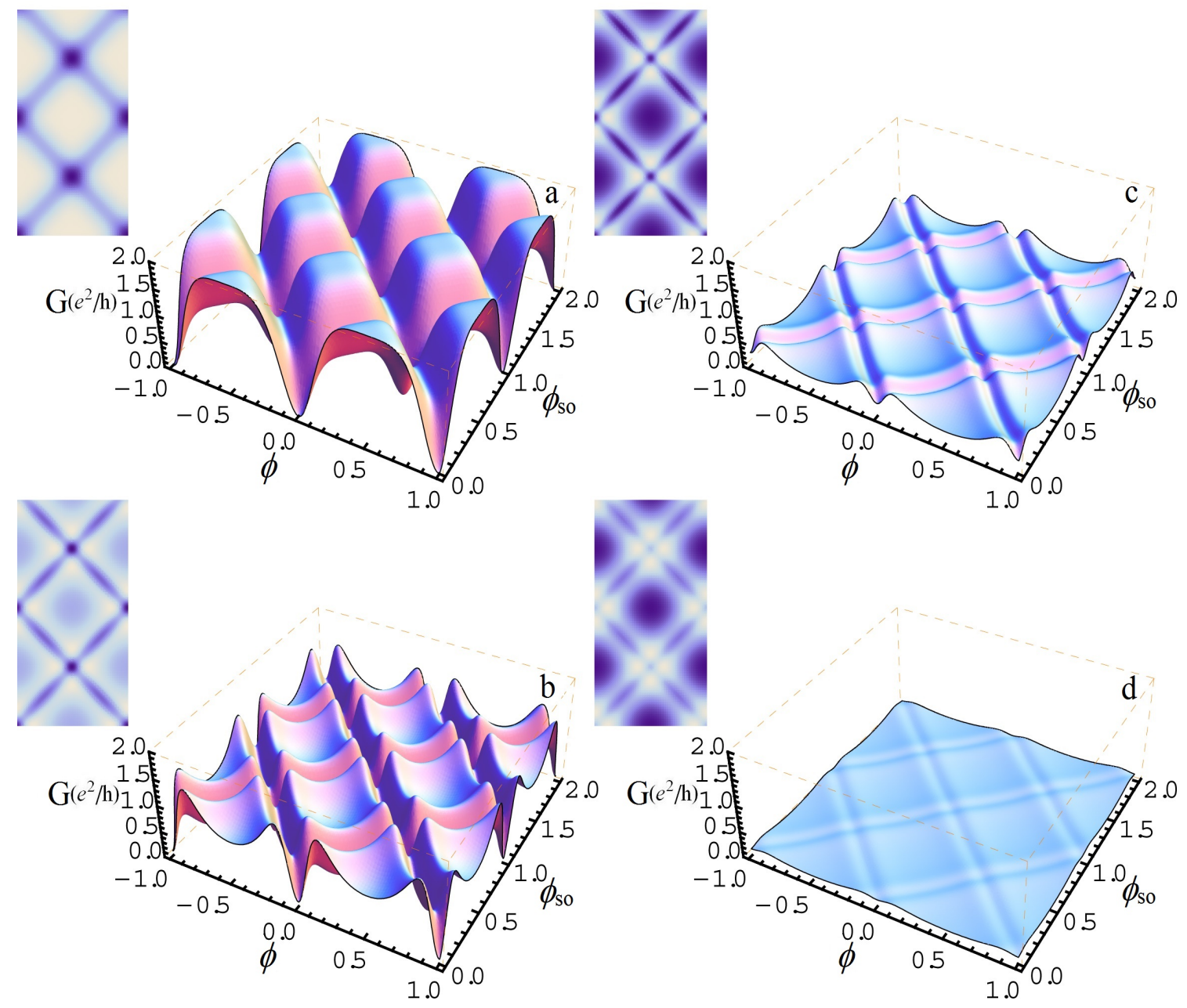

FIG. 7: (Color online ) Conductance oscillations as a function of the $\mathrm{AB}$ phase $\phi$ and $\mathrm{AC}$ phase $\phi_{\mathrm{SO}}$. The transmission coefficients used at the junctions $\left(\mathrm{QPC}_{1,2}\right)$ and at the scatter $\left(\mathrm{QPC}_{3}\right)$, in the panels $a, b, c$, and $d$; correspond to: $T_{o} \equiv T_{s}=1,3 / 4,1 / 2$, and $1 / 3$, respectively. Since $T_{o(s)}$ depend also on $\hbar \omega_{j}$, these are varied simultaneously as the AB-phase $\phi$ is tuned in order to have an uniform transmission probability of the QPCs as $\phi$ changes. The insets show the same information in a density-map format for $\phi$ ranging in the interval $[-1,0]$ which corresponds to a magnetic field interval between -21 to $0 \mathrm{mT}$.

the robustness of the periodic pattern of $G$ are still visible even though the transmission of the QPCs are strongly reduced up to 1/3 [see Fig.7(b- $d)$ and insets].

Thus, just by controlling simultaneously the aperture of $\mathrm{QPC}_{1,2,3}$ we can achieve three qualitative different physical configurations for conductance response of the $\mathrm{QR}$ device as 

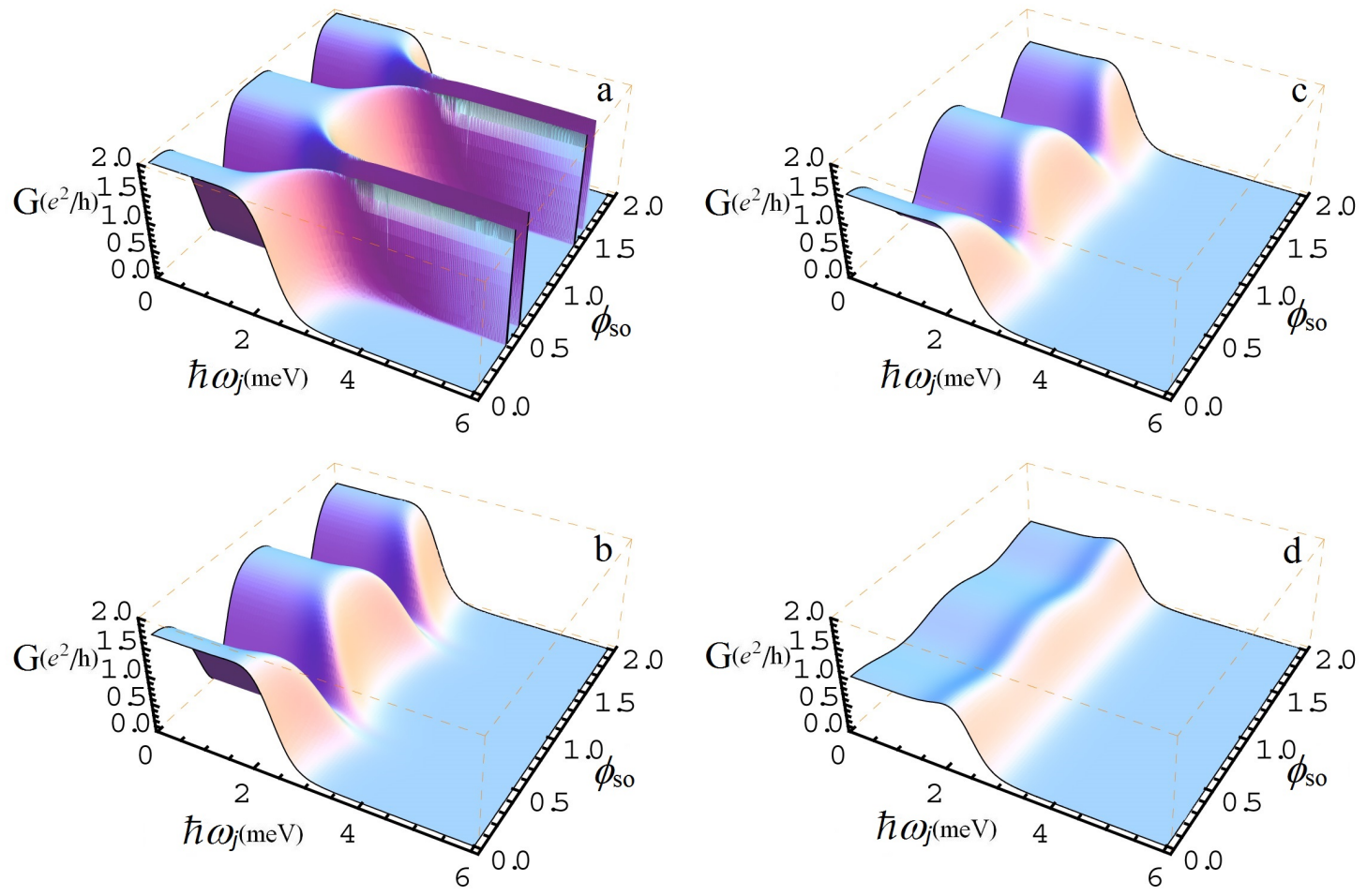

FIG. 8: (Color online) Behavior of the conductance as a function of $\hbar \omega_{j}$ and AC phase $\phi_{\text {So }}$. We have fixed the $\mathrm{AB}$ phase $\phi=0.5(B=10 \mathrm{mT})$. The transmission coefficients used at the scatter $\left(\mathrm{QPC}_{3}\right)$, in the panels a, b, c, and d correspond to $T_{s}=1,1 / 2,1 / 4$ and $10^{-3}$, respectively.

described in panels $a, b$ and $c$ of Fig.7. Notice also that due the possible control of the Aharanov-Casher phase by the electrical tuning of the Rashba spin-orbit coupling together with the gate control of the confinement of the QPCs, leads to the possibility of switching $\mathrm{ON}$ and $\mathrm{OFF}$ the current through the QR device, even in the absence of magnetic field $(\phi=0)$. A similar scenario can be achieved by, on the contrary, fixing the Rashba coupling and varying the QPCs confinement strength, also at zero magnetic field. This constitutes an advantageous feature over those QR devices that rely on a magnetic flux to manifest a similar behavior. This quantum effect suggests the possibility of using our QR device proposal as a logic gate 39] and in appealing spintronics applications devices whose performance can be manipulated by adjusting gate voltages only.

Finally, in Fig. 8 we study the behavior of the conductance quantum oscillations as a function of $\hbar \omega_{j}$ and $\mathrm{AC}$ phase $\phi_{\mathrm{So}}$ for several values of the transmission coefficients $T_{s}$ for the upper-arm $\mathrm{QPC}_{3}$. Cases with $T_{s}=1,1 / 2,1 / 4$ and $10^{-3}$ are considered in panels $a, b, c$ 
and $d$, respectively. It is clear that the persistent resonances appearing for $\hbar \omega_{j} \gtrsim 3 \mathrm{meV}$ in Fig.8 a vanish completely by closing $\mathrm{QPC}_{3}$, see Fig.8b and $c$ for instance. Such effect respond to the disappereance of the spectrum of the quasi-stationary states of the close $\mathrm{QR}$ as soon a sizable scatterer is present in the path way of the circling electrons, which in turn destroys quantum interference. Notice that despite the maximum opacity $\left(T_{s}=10^{-3}\right)$ imposed in the $\mathrm{QPC}_{3}(\mathrm{Fig}, 8 \mathrm{~d})$ the conductance do not vanish completely but rather fluctuates around $G \approx e^{2} / h$, in sharp contrast with the phenomenology observed in Fig.7 $a / d$ in which the QPCs at the lead-to-ring-junctions are essentially blocked instead. In the former case the coherent quantum interference of electrons is completely suppressed.

These features described above comprises yet another distinctive behavior of $G$ profiles in the QR device proposed here. Indeed, depending on the value of $T_{s}$, and by considering the full axis $0 \leq \hbar \omega_{j} \leq 6 \mathrm{meV}$, one can obviously achieve three different configurations for $G$. Namely, as shown in Fig, 8 a maximum fluctuations of $G$ can be attained in conjunction with very sharp resonances, while e.g. in panel $(c)$ the wide oscillations are diminished in amplitude and the sharp resonance vanishes. The third distinctive pattern is seen in Fig $8 d$, where the oscillations are strongly suppressed due the strong confinement strength in $\mathrm{QPC}_{3}$ which in turn inhibit spin quantum interference.

\section{SUMMARY}

We have examined the electron spin-interference and the coherent electronic spintransport through a proposed semiconductor QR-device with three embedded QPCs in the presence of Rashba spin-orbit interaction. A suitable model for the QPCs based in twodimensional saddle point potentials and known transmission coefficients is employed. In contrast to previous theoretical studies, the quantum point contacts are included at the inlet and outlet of the QR, which can be used to control the transmission in different parts of the structure and give us the freedom to vary electrically the interference pattern of the QR. Using the $\mathbb{S}$-matrix formalism we have derived a closed analytical expression for the total transmitted amplitude through the QR-device that incorporates the confinement strength, external magnetic field and Rashba spin-orbit coupling in the same footing. We also derive the conditions for resonances and anti-resonances of the conductance of the QR. Such for-

mulas for the spin-conductance holds in general, independent of the transmission probability 
model considered for the QPCs. Our theoretical modeling for the Landauer conductance readily reproduce the expected periodic-flux conductance effects reported in the literature in the absence of QPCs; with and without Rashba coupling.

When considering the influence of the scattering at the QPCs, we found that the electron spin interference in the QR-device is very sensitive to the QPC confinement strengths, offering new features amenable to control the electron spin-transport and the quantum spininterference in the QR. For instance we found that, $(i)$ the oscillatory pattern and the spin interference in the QR can be controlled to great extend by varying the lead-to-ring transmission probabilities (through the confinement strengths of the QPCs), ( $i i)$ the conductance oscillations exhibit a crossover to well-defined sharp resonance behavior as the QPCs confinement is increased, ( iii) the simultaneous electrical control of the aperture of all QPCs yields three remarkably different behavior of the conductance response as a function of the Aharanov-Bohm and Aharanov-Casher phases, and ( $i v)$ also distinctive behavior of the conductance results by varying the aperture of the upper-arm QPC and the Rashba spin-orbit coupling.

The new features predicted in the proposed QR-device may be of utility to implement spintronic logic gate devices based on quantum interference and whose function can be manipulated using gate voltages only. The theoretical modeling is flexible to incorporate more scatterers in the QR, Dresselhaus type of spin-orbit interaction[43], spin-flip mixing and asymmetric injection/detection of electrons.

\section{Acknowledgments}

One of the authors (L.D-C) is grateful to the Visiting Academic Program of the UIAMéxico and acknowledges the hospitality and facilities offer by CNyN-UNAM, Ensenada, México where part of this research was executed. This work was partially supported by project PAPIIT-DGAPA (UNAM) No. IN109911.

\section{Appendix A: Derivation of the total transmission amplitude}

In this appendix we derive Eq.(28) for the scattering amplitude $\alpha_{2 \sigma}^{\prime}$ that characterize the probability of the emergent electrons to the right hand side of the QR device. 
First we realize that with the aid of the expressions (20), 21) and (25), it is possible to establish the useful relationship

$$
\left[\begin{array}{c}
\beta_{2 \sigma} \\
\beta_{2 \sigma}^{\prime}
\end{array}\right]=\boldsymbol{\Phi}_{u}^{\sigma} \cdot \mathbb{T}_{u} \cdot \boldsymbol{\Phi}_{u}^{\sigma}\left[\begin{array}{c}
\beta_{1 \sigma}^{\prime} \\
\beta_{1 \sigma}
\end{array}\right]
$$

then consider (24) in the compact form

$$
\left[\begin{array}{c}
\gamma_{1 \sigma} \\
\gamma_{1 \sigma}^{\prime}
\end{array}\right]=\boldsymbol{\Phi}_{l}^{\sigma} \cdot \mathbb{T}_{l}\left[\begin{array}{c}
\gamma_{2 \sigma}^{\prime} \\
\gamma_{2 \sigma}
\end{array}\right]
$$

and assume electron incidence from the left lead only, with an unitary incident current flux, i.e. $\alpha_{1 \sigma}=1$ and $\alpha_{2 \sigma}=0$. By using (A1) and (A2) together with (20)-(23), (25) and (28) it is possible to connect the amplitudes of the ingoing/outgoing waves arriving/leaving the $\mathrm{QPC}_{1}$ and $\mathrm{QPC}_{2}$ in the $\mathrm{QR}$ by writing

$$
\left[\begin{array}{c}
\beta_{1 \sigma}^{\prime} \\
\beta_{1 \sigma}
\end{array}\right]=-\frac{\sqrt{2} T_{s}^{1 / 2}}{\left(R_{s}^{1 / 2}+\lambda\right)}\left[\begin{array}{c}
\lambda \\
-1
\end{array}\right]+\boldsymbol{t}_{j}\left[\begin{array}{c}
\gamma_{1 \sigma} \\
\gamma_{1 \sigma}^{\prime}
\end{array}\right]
$$

whereas

$$
\left[\begin{array}{c}
\gamma_{2 \sigma}^{\prime} \\
\gamma_{2 \sigma}
\end{array}\right]=\boldsymbol{t}_{j}\left[\begin{array}{c}
\beta_{2 \sigma} \\
\beta_{2 \sigma}^{\prime}
\end{array}\right]
$$

being $\lambda=\mathrm{c}-\mathrm{b}= \pm 1$. Next, by following a similar procedure of Ref.27 we have derived

$$
\boldsymbol{t}_{j 1} \equiv \boldsymbol{t}_{j 2}=\boldsymbol{t}_{j}=\frac{1}{\mathrm{c}}\left(\begin{array}{cc}
\mathrm{c}^{2}-\mathrm{b}^{2} & \mathrm{~b} \\
-\mathrm{b} & 1
\end{array}\right)
$$

where $\boldsymbol{t}_{j 1}$, and $\boldsymbol{t}_{j 2}$ represents the effective transmission amplitudes matrices at the left and right lead-to-ring junctions, respectively. From Eqs. (A1), (A2) and (A3) we obtain

$$
\left[\begin{array}{c}
\beta_{1 \sigma}^{\prime} \\
\beta_{1 \sigma}
\end{array}\right]=-\frac{\sqrt{2} T_{s}^{1 / 2}}{\left(R_{s}^{1 / 2}+\lambda\right)}\left[\begin{array}{c}
\lambda \\
-1
\end{array}\right]+\boldsymbol{t}_{j} \boldsymbol{\Phi}_{l}^{\sigma} \mathbb{T}_{l} \boldsymbol{t}_{j}\left[\begin{array}{c}
\beta_{2 \sigma} \\
\beta_{2 \sigma}^{\prime}
\end{array}\right]
$$

Similarly, we can conveniently write the amplitude of the outgoing wave to the right lead as follows

$$
\left[\begin{array}{c}
\alpha_{2 \sigma}^{\prime} \\
0
\end{array}\right]=-\left(\frac{\sqrt{2} T_{s}^{1 / 2}}{R_{28}^{1 / 2}+\lambda}\right) \lambda\left[\begin{array}{c}
\beta_{2 \sigma} \\
\beta_{2 \sigma}^{\prime}
\end{array}\right]
$$


where we have used (29)

$$
\boldsymbol{\lambda}=\left(\begin{array}{ll}
\lambda & 1 \\
0 & 0
\end{array}\right)
$$

in which either value of $\lambda( \pm 1)$ gives a possible solution of the scattering problem. We can now use (A6) and (A7) we arrive to the column vector

$$
\left[\begin{array}{c}
\beta_{2 \sigma} \\
\beta_{2 \sigma}^{\prime}
\end{array}\right]=\left(\frac{\sqrt{2} T_{s}^{1 / 2}}{R_{s}^{1 / 2}+\lambda}\right)\left(\mathbb{P}^{\sigma}\right)^{-1} \boldsymbol{\Phi}_{u}^{\sigma} \mathbb{T}_{u} \boldsymbol{\Phi}_{u}^{\sigma}\left[\begin{array}{c}
\lambda \\
-1
\end{array}\right],
$$

in which we have introduced the $(2 \times 2)$ matrix $\mathbb{P}^{\sigma}=\boldsymbol{\Phi}_{u}^{\sigma} \mathbb{T}_{u} \boldsymbol{\Phi}_{u}^{\sigma} \boldsymbol{t}_{j 1} \boldsymbol{\Phi}_{l} \mathbb{T}_{l} \boldsymbol{t}_{j 2}-\boldsymbol{I}_{2}$. Finally, inserting (A9) into (A7) we get (28)

$$
\left[\begin{array}{c}
\alpha_{2 \sigma}^{\prime} \\
0
\end{array}\right]=\frac{2 T_{s}}{\left(R_{s}^{1 / 2}+\lambda\right)^{2}} \boldsymbol{\lambda}\left(\mathbb{P}^{\sigma}\right)^{-1} \boldsymbol{\Phi}_{u}^{\sigma} \mathbb{T}_{u} \boldsymbol{\Phi}_{u}^{\sigma}\left[\begin{array}{c}
-\lambda \\
1
\end{array}\right],
$$

which provides a closed form for the total transmitted amplitude $\alpha_{2 \sigma}^{\prime}$ of the outgoing electrons at the right lead in terms of the transmission amplitudes of the QPCs and all the quantum phases involved.

[1] I. Zutic, J. Fabian, and S. Das Sarma, Rev. Mod. Phys. 76, 323 (2004).

[2] Y. Aharonov and D. Bohm, Phys. Rev. 115, 485 (1959).

[3] Y. Aharonov and A. Casher, Phys. Rev. Lett.53, 319 (1984).

[4] J. Nitta, T. Akasaki, H. Takayanagi, and T. Enoki, Phys. Rev. Lett. 78, 1335 (1997); T. Koga, J. Nitta, T. Akasaki, and H. Takayanagi, ibid. 89, 046801 (2002).

[5] G. Engels, J. Lange, Th. Schäpers, and H. Lüth, Phys. Rev. B 55, R1958 (1997).

[6] Th. Schäpers, G. Engels, J. Lamge, Th. Klocke, M. Hollfelder, and H. Lüth, J. Appl. Phys. 83, 4324 (1998).

[7] X. F. Wang and P. Vasilopoulos, Phys. Rev. B 72, 165336 (2005).

[8] J. Nitta, T. Bergstein, Y. Kunihashi, and M. Kohda, J. Appl. Phys. 105, 122402 (2009).

[9] R.A. Webb, S. Washburn, C.P. Umbach and R. B. Laibowitz, Phys. Rev. Lett. 54,2696 (1985). 
[10] A. Cimmino, G.I. Opat, A.G. Klein, H. Kaiser, S.A. Werner, M. Arif, and R. Clothier, Phys. Rev. Lett. 53, 380 (1989).

[11] M. König, A. Tschetschetkin, E.M. Hankiewicz, J. Sinova, V. Hock, V. Daumer, M. Schäfer, C.R. Becker, H. Buhmann and L.W. Molenkamp, Phys. Rev. Lett. 96, 076804 (2006).

[12] T. Bergsten, T. Kobayashi, Y. Sekine, and J. Nitta, Phys. Rev. Lett. 97, 196803 (2006).

[13] E.I. Rashba, Sov. Phys. Solid State 2, 1109 (1960): Y.A. Bychkov and E.I. Rashba, J. Phys. C 17, 6039 (1984).

[14] M. V. Berry, Proc. R. Soc. London, Ser. A 392, 45 (1984).

[15] D. Frustaglia and K. Richter Phys. Rev. B 69, 235310 (2004).

[16] D. Bercioux, Di. Frustaglia and M. Governale, Phys. Rev. B 72, 113310 (2005).

[17] F. Nagasawa, J. Takagi, Y. Kunihashi, M. Kohda, and J. Nitta, Phys. Rev. Lett. 108, 086801 (2012).

[18] T.-Z. Qian and Z.-B.Su, Phys Rev. Lett. 72, 2311 (1994).

[19] K. Richter, Physics 5, 22 (2012).

[20] J. Nitta, F.E. Meijer, and H. Takayanagi, Appl. Phys. Lett. 75, 695 (1999).

[21] A. G. Aronov and Y. B. Lyanda-Geller, Phys. Rev. Lett. 70, 343 (1993).

[22] A.A. Kiselev and K. W. Kim, J. Appl. Phys. 94, 4001 (2003).

[23] I. A. Shelykh, N. G. Galkin, and N. T. Bagraev, Phys. Rev. B 72, 235316 (2005).

[24] I. A. Shelykh, N. T. Bagraev, N. G. Galkin, L. E. Klyachkin, Phys. Rev. B 71, 113311 (2005).

[25] M. Büttiker, Y. Imry and M. Ya. Azbel, Phys. Rev. A 30, 1982 (1984).

[26] I. A. Shelykh, N. T. Bagraev, N. G. Galkin, L. E. Klyachkin, Phys. Rev. B 71, 113311 (2005).

[27] P. Vasilopoulos, O. Kálmán, F. M. Peeters, and G. Benedict, Phys. Rev. B 75, 035304 (2007).

[28] H. A. Fertig and B. I. Halperin, Phys. Rev. B 36, 7969 (1987).

[29] F.E. Meijer, A.F. Morpurgo, and T.M. Klapwijk, Phys. Rev. B 66, 033107 (2002).

[30] E. N. Bulgakov, A. F. Sadreev Phys. Rev. B 66, 075331 (2002).

[31] N. T. Bagraev, N. G. Galkin, W. Gelhoff, I.E. Klyachkin, A.M. Malyarenko, and I. A. Shelykh, J. Phys.: Condens. Matter 18, L567 (2006).

[32] R. Landauer, J. Phys. Condens. Matter 1, 8099 (1989).

[33] M. Büttiker, Phys. Rev. B 41, 7906 (1990).

[34] H. van Houten, L.W. Molenkamp, C.W.J. Beenakker, C.T. Foxon, Semicond. Sci. Technol. 7, B215 (1992). 
[35] M.J. Gilbert and J. Bird, Appl. Phys. Lett. 77, 1050 (2000).

[36] Y. Sato, T. Kita, S. Goza, and S. Yamada, J. Appl. Phys. 81, 8017 (2001).

[37] J. Nitta, and T. Koga, J. Supercond. 16, 689 (2003).

[38] B. Molnár, F. M. Peeters and P. Vasilopoulos, Phys. Rev. B 69, 155335 (2004).

[39] P. Foldi, B. Molnár, M. G. Benedict, and F. M. Peeters, Phys. Rev. B, 71, 033309 (2005).

[40] E. Zipper, M. Kurpas and M.M. Maska, New Journal of Physics 14, 093029 (2012).

[41] S. Amasha, K. MacLean, I. P. Radu, D. M. Zumbühl, M. A. Kastner, M. P. Hanson, and A. C. Gossard, Phys. Rev. Lett. 100, 046803 (2008).

[42] M. Kroutva, Y. Ducommun, D. Heiss, M. Bichler, D. Schuh, G. Abstreiter, and J. Finley, Nature 432, 81 (2004).

[43] Kh. Shakouri, B. Szafran, M. Esmaeilzadeh, and F. M. Peeters, Phys. Rev. B 85, 165314 (2012). 